\title{
Article \\ Photoacoustic Imaging of Human Vasculature Using LED versus Laser Illumination: A Comparison Study on Tissue Phantoms and In Vivo Humans
}

\author{
Sumit Agrawal 1,+(D), Mithun Kuniyil Ajith Singh ${ }^{2,+}{ }^{1}$, Kerrick Johnstonbaugh ${ }^{1}$, David C. Han ${ }^{3,4}$, \\ Colette R. Pameijer ${ }^{4}$ and Sri-Rajasekhar Kothapalli 1,5,6,*(D)
}

check for updates

Citation: Agrawal, S.; Kuniyil Ajith Singh, M.; Johnstonbaugh, K.; C. Han, D.; R. Pameijer, C.; Kothapalli, S.-R. Photoacoustic Imaging of Human Vasculature Using LED versus Laser Illumination: A Comparison Study on Tissue Phantoms and In Vivo Humans. Sensors 2021, 21, 424. https://doi.org/10.3390/s21020424

Received: 16 December 2020 Accepted: 6 January 2021 Published: 9 January 2021

Publisher's Note: MDPI stays neutral with regard to jurisdictional clai$\mathrm{ms}$ in published maps and institutional affiliations.

Copyright: $(2021$ by the authors. Licensee MDPI, Basel, Switzerland. This article is an open access article distributed under the terms and conditions of the Creative Commons Attribution (CC BY) license (https:// creativecommons.org/licenses/by/ $4.0 /)$.
1 Department of Biomedical Engineering, Pennsylvania State University, University Park, State College, PA 16802, USA; sua347@psu.edu (S.A.); kjohnstonbaugh97@gmail.com (K.J.)

2 Research \& Business Development Division, CYBERDYNE INC, Cambridge Innovation Center, 3013 AK Rotterdam, The Netherlands; mithun_ajith@cyberdyne.jp

3 Department of Surgery, Penn State Heart and Vascular Institute, Hershey, PA 16802, USA; dch15@psu.edu

4 Penn State Hershey College of Medicine and Milton S. Hershey Medical Center, Hershey, PA 17033, USA; cpameijer@pennstatehealth.psu.edu

5 Penn State Cancer Institute, Pennsylvania State University, Hershey, PA 17033, USA

6 Graduate Program in Acoustics, Pennsylvania State University, University Park, State College, PA 16802, USA

* Correspondence: srkothapalli@psu.edu

+ These authors contributed equally to this work.

\begin{abstract}
Vascular diseases are becoming an epidemic with an increasing aging population and increases in obesity and type II diabetes. Point-of-care (POC) diagnosis and monitoring of vascular diseases is an unmet medical need. Photoacoustic imaging (PAI) provides label-free multiparametric information of deep vasculature based on strong absorption of light photons by hemoglobin molecules. However, conventional PAI systems use bulky nanosecond lasers which hinders POC applications. Recently, light-emitting diodes (LEDs) have emerged as cost-effective and portable optical sources for the PAI of living subjects. However, state-of-art LED arrays carry significantly lower optical energy ( $<0.5 \mathrm{~mJ} /$ pulse) and high pulse repetition frequencies (PRFs) $(4 \mathrm{KHz})$ compared to the high-power laser sources $(100 \mathrm{~mJ} /$ pulse) with low PRFs of $10 \mathrm{~Hz}$. Given these tradeoffs between portability, cost, optical energy and frame rate, this work systematically studies the deep tissue PAI performance of LED and laser illuminations to help select a suitable source for a given biomedical application. To draw a fair comparison, we developed a fiberoptic array that delivers laser illumination similar to the LED array and uses the same ultrasound transducer and data acquisition platform for PAI with these two illuminations. Several controlled studies on tissue phantoms demonstrated that portable LED arrays with high frame averaging show higher signal-to-noise ratios (SNRs) of up to $30 \mathrm{~mm}$ depth, and the high-energy laser source was found to be more effective for imaging depths greater than $30 \mathrm{~mm}$ at similar frame rates. Label-free in vivo imaging of human hand vasculature studies further confirmed that the vascular contrast from LED-PAI is similar to laser-PAI for up to $2 \mathrm{~cm}$ depths. Therefore, LED-PAI systems have strong potential to be a mobile health care technology for diagnosing vascular diseases such as peripheral arterial disease and stroke in POC and resource poor settings.
\end{abstract}

Keywords: deep tissue imaging; hemangioma; laser; light-emitting diodes (LED); mobile health; peripheral arterial disease; photoacoustic imaging; stroke; vascular malformations

\section{Introduction}

Vascular diseases are the leading cause of death worldwide. Some common vascular diseases include cardiovascular disease, stroke and peripheral artery disease (PAD) [1-3]. Many of these vascular diseases need point-of-care (POC) diagnosis and monitoring using 
nonionizing, noninvasive and cost-effective approaches. Although Doppler ultrasound meets all these requirements, it only maps blood flow, which is operator dependent and influenced by motion artifacts, resulting in limited sensitivity and specificity to detect the disease in its early stage [4]. A POC technique that provides direct label-free molecular and functional information of vasculature is needed to reliably detect and monitor vascular diseases [5,6].

Photoacoustic imaging (PAI) is a hybrid imaging modality that provides rich optical spectroscopic contrast at ultrasonic penetration depths and resolutions [7]. Over the past two decades, PAI has emerged as a promising tool for label-free imaging of individual blood vessels [8,9], detection of angiogenesis [10] and has also helped in extracting several physiologically relevant parameters such as blood oxygen saturation [11,12] and changes in the blood volume [13], which are vital for monitoring disease progression [14,15]. The basic mechanism of PAI includes a nanosecond pulsed optical excitation that illuminates the biological subject. The light absorbing molecules inside the tissue undergo thermoelastic expansion and generate broadband acoustic waves which are subsequently detected using conventional ultrasound (US) detectors [7]. Biomedical PAI mainly capitalizes on the intrinsic absorbers present in human tissue [8], such as oxy- and deoxyhemoglobin [10,11], melanin [16], lipids [17,18], water [19], RNA and DNA [20], as each of these exhibits a characteristic absorption spectrum. However, if the spectral contrast from these intrinsic chromophores is not sufficient to reveal the disease, a wide range of extrinsic contrast agents [21-25] can be functionalized to target different diseased biomarkers to increase molecular sensitivity and specificity.

While ultrasound and certain optical technologies are available in the size of a mobile phone [26,27], PAI systems still have to reach that level of portability. Conventional PAI systems employ bulky class-IV laser sources (100 mJ/pulse) and data acquisition systems to increase the peak imaging performance [28-31]. Such lasers not only increase the system cost and footprint but also carry a high risk of class-IV exposure. However, to translate PAI technology to POC clinical applications and to resource-limited settings, a significant reduction in both cost and size is required. To address this challenge, several cost-effective alternatives for both the optical excitation [32-35] and the ultrasound detection [36-39] components have been explored, including for wearable applications $[40,41]$.

Recently, low-power laser diodes [42,43] and light-emitting-diodes (LEDs) [44] have been proposed as alternatives to laser sources. Specifically, pertaining to their noncoherent nature, LEDs carry a huge potential to be the safe and cost-effective alternative illumination sources for PAI $[43,44]$. However, compared to the class-IV lasers, LED arrays carry much less optical energy (i.e., order of $100^{\prime} \mathrm{s} \mu \mathrm{J}$ ) per pulse, and thus their PAI capabilities as a function of imaging depth need to be studied in detail to employ a suitable optical source for a given POC application $[45,46]$.

To enhance the performance of LEDs, an arrayed arrangement of LED elements was developed $[47,48]$, thereby increasing the pulse energies from a few $\mu \mathrm{J}$ to hundreds of $\mu \mathrm{J}$. In addition to this, higher pulse repetition frequency (PRF) rates (i.e., $\sim 4 \mathrm{KHz}$ ) of the LEDs allowed a sufficient PA frame averaging which led to significant signal-to-noise ratio (SNR) improvements for deep tissue targets [46]. These LED array B-mode PAUS [47,48] and tomographic imaging $[49,50]$ setups have been demonstrated using several preclinical small animals [51,52] and in vivo human imaging studies [53-55].

To date, there is no study that quantitatively compares the PAI performance of LEDs and laser illumination head-to-head. Given these tradeoffs between portability, cost, optical energy and frame rate, this work systematically studies the deep tissue PAI performance of LED and laser excitation to help select a suitable source for a given biomedical application. First, a setup for sequentially performing PAI with these two optical illuminations has been developed. Controlled studies on different tissue phantoms have been performed for detailed evaluation of the imaging performance. Further, in vivo human hand vasculature imaging in 2-D and 3-D was performed using these two optical sources. The rest of the paper is organized as follows. Section 2 describes our proposed setup for comparing the 
two sources. Comparison studies including tissue-mimicking phantoms and the in vivo human imaging are presented in Section 3. Section 4 provides a detailed discussion of the results.

\section{Materials and Methods}

In this section, a detailed description of the experimental setup for studying the PAI capabilities of high-power laser and LED array sources is presented. First, the commercial LED array-based B-mode PA and ultrasound (US) imaging system (AcousticX, Cyberdyne Inc., Ibaraki, Japan), referred to in this paper as LED-PAUS, is presented in Section 2.1 and then the modifications performed to use the same AcousticX data acquisition system for laser-illumination-based PA, referred to as laser-PAUS, without interrupting parameters of the imaging system, are presented in Section 2.2.

\subsection{LED Array-Based US/PA (LED-PAUS) Imaging System Description}

Conventionally, a high-power and bulky laser source is employed for most B-mode PA and US systems [28,29]. The commercial LED-PAUS system, as shown in Figure 1, consists of a host controller (Figure 1a), data acquisition hardware (Figure 1b) and an interactive graphical user interface shown on the display in Figure 1d. Here, a linear ultrasound probe is sandwiched between two LED arrays to capture interleaved B-mode PA and US images, as shown in Figure 1e. Each LED array consists of four rows of 36 LED elements $(1 \times 1 \mathrm{~mm}$ size). The LED arrays used in this study consist of $850 \mathrm{~nm}$ LED elements and each array provides an output energy of $200 \mu \mathrm{J} /$ pulse. The excitation pulse widths for each of these LED arrays can be controlled with the software in the range of 30 to $150 \mathrm{~ns}$. For all the experiments in this study, a pulse width of 70 ns was used that offered optimum energy at $850 \mathrm{~nm}$ [56]. The optical illumination profile achieved with the two $850 \mathrm{~nm}$ LED arrays operated with pulse widths of $70 \mathrm{~ns}$ is shown in Figure $1 \mathrm{~g}$. The shape of the beam falling on skin is approximately a rectangle with an area of $9 \mathrm{~cm}^{2}(5$ by $1.8 \mathrm{~cm}$ ), leading to an incident optical fluence of $0.044 \mathrm{~mJ} / \mathrm{cm}^{2}$, considering $400 \mu \mathrm{J}$ total energy per pulse with the two LED arrays. The pulse repetition frequency (PRF) of these LEDs can also be controlled in the range of 1 to $4 \mathrm{KHz}$ allowing multiple averaging options leading to different frame rates. To assess the effect of changing the frame rate over the PA image quality, several different combinations of PRF and frame averaging are used in this study, as discussed in the subsequent sections. The US probe used in this study is a 128-element linear US array with a pitch of $0.3 \mathrm{~mm}$, center frequency of $7 \mathrm{MHz}$, elevational focus of $15 \mathrm{~mm}$ and a measured $6 \mathrm{~dB}$ bandwidth of $75 \%$. The system provides PA and US acquisition sampling rates of 40 and $20 \mathrm{MHz}$, respectively.

\subsection{Experimental Setup for Comparing LED-PAUS and Laser-PAUS}

Figure 1 presents the overall experimental setup developed to compare the performance of LED-PAUS and laser-PAUS imaging. The LED-PAUS system, described in Section 2.1, was adapted for laser-PAUS imaging. In laser-PAUS, a portable optical parametric oscillator (OPO) laser source (Phocus Mobile, Opotek, Inc., Carlsbad, CA, USA), tunable in the range of 690-950 nm, shown in Figure 1c, provided the laser illumination. The laser has a fixed pulse width of 5-7 ns, a fixed PRF of $10 \mathrm{~Hz}$ and an output energy of $140 \mathrm{~mJ}$ per pulse at $750 \mathrm{~nm}$. For this study, $850 \mathrm{~nm}$ wavelength laser illumination was used with the optical energy tuned down to $40 \mathrm{~mJ} /$ pulse. Light output from the laser was coupled to a $2 \mathrm{~m}$ long custom designed optical fiber bundle (Fiberoptic System Inc., Simi Valley, CA, USA). The fiber had a fused end with a diameter of $6.5 \mathrm{~mm}$ that entered into the tunable output port of the laser, as shown in Figure 1c. The distal end of this fiber was split into twenty smaller (1.45 $\mathrm{mm}$ inner diameter) fibers with numerical apertures of 0.55 , each sharing equal optical energy. Ten out of these twenty fibers were inserted into each of the two custom designed 3-D printed fiber holders attached each side of the US probe, as shown in Figure 1f. This design allowed laser illumination similar to the case of LED arrays, in terms of the illumination angle and the overall geometry around the 
US probe. Further, to achieve a uniform illumination profile on the tissue surface, two glass diffusers (N-BK7 Ground Glass Diffuser 1500 Grit, Thorlabs Inc., Newton, NJ, USA) were attached at the output end of the fiber holders, as shown in Figure 1f. The resulting laser-illumination profile at $850 \mathrm{~nm}$ is shown in Figure 1h. The shape of the beam falling on skin is approximately a rectangle with an area of $6 \mathrm{~cm}^{2}(5 \mathrm{by} 1.2 \mathrm{~cm})$, leading to an incident optical fluence of $6.66 \mathrm{~mJ} / \mathrm{cm}^{2}$, considering $40 \mathrm{~mJ}$ total energy per pulse with the laser illumination. In our experimental setup, switching from LED arrays to a laser fiber setup and vice versa was convenient and did not disturb the US probe as well as the imaging subject. Figure 1i,j show the heads of LED-PAUS and laser-PAUS systems for acquiring respective B-mode PA and US images of human hand vasculature.
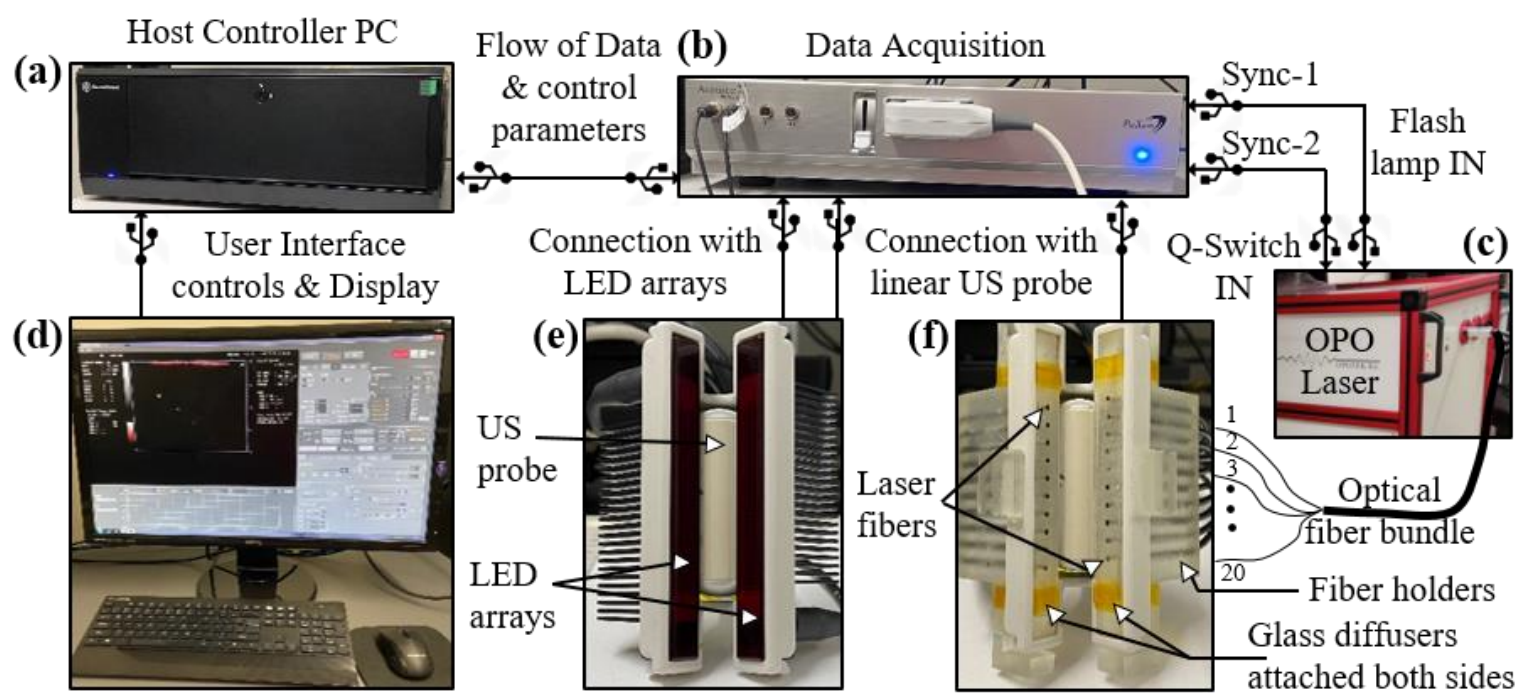

(g)

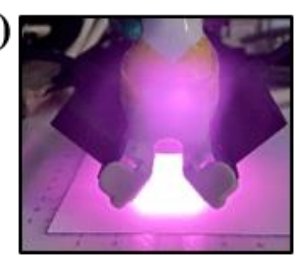

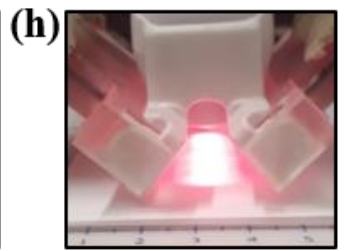

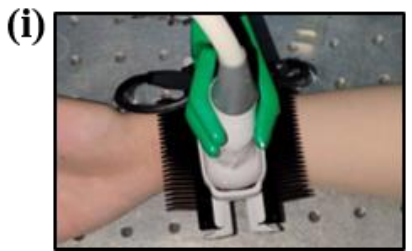

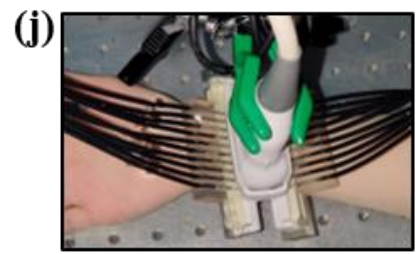

Figure 1. Description of the experimental setup designed for comparing light-emitting diode (LED)-based and highpower laser-based photoacoustic (PA) and ultrasound (US) imaging. The setup consists of the following key components: Commercial B-mode LED-PAUS system (AcousticX, Cyberdyne Inc., Ibaraki, Japan) with (a) a host controller PC and (b) data acquisition hardware. (c) A portable high-power laser (Phocus Mobile, Opotek Inc., Carlsbad, CA, USA) with its output coupled to the input end of an optical fiber bundle. The fiber bundle is split into twenty smaller fibers at the distal end. (d) Computer display: displays B-mode US (grayscale), PA (red scale), and coregistered US + PA (overlaid red PA on gray US). The interface also enables switching between LED and laser operation. (e) Arrangement of two $850 \mathrm{~nm}$ LED arrays around the US probe. (f) Arrangement of twenty laser fibers inserted into the two fiber holders around the US probe. Two glass diffusers attached at the fiber output ends to provide uniform laser illumination on the tissue surface. Optical illumination profile achieved with (g) two LED array sources and (h) laser source. (i,j) Pictures of a human wrist under imaging with the LED and laser arrangements, respectively.

\section{Validation Experiments and Results}

In this section, an extensive evaluation of the imaging performance of low-power LED-PAUS and high-power laser-PAUS systems is presented with the help of rigorous SNR and resolution studies on several tissue phantoms, in vivo imaging of the human wrist and in vivo 3-D vasculature mapping of the human forearm. 


\subsection{Photoacoustic Imaging Comparison of LED Arrays and Laser Source Using a Scattering Phantom}

In this subsection, a controlled study evaluating the deep tissue PAI capabilities of lowpower LED arrays and a high-power laser source is presented. An acrylic tank with four holes was fabricated and a pencil lead with a diameter of $0.5 \mathrm{~mm}$ was inserted into each hole. The tank was filled with an intralipid solution to mimic an optical reduced scattering coefficient $\left(\mu_{s}^{\prime}\right)$ of $20 \mathrm{~cm}^{-1}$. Considering an approximate optical absorption coefficient $\left(\mu_{a}\right)$ of 0.05 to $0.1 \mathrm{~cm}^{-1}$, the effective attenuation coefficient $\left(\mu_{e f f}\right)$ of the intralipid phantom was in the range of 1.73 to $2.45 \mathrm{~cm}^{-1}\left(\mu_{e f f}=\sqrt{3 \mu_{a}\left(\mu_{a}+\mu_{s}^{\prime}\right)}\right)$. Figure 2a shows the schematic of the experimental setup with four pencil lead targets diagonally arranged along the depth of imaging inside the intralipid solution. The measured depth of these four pencil leads from the surface of the US transducer were 15, 23, 28, and $34 \mathrm{~mm}$, respectively.

The laser-illumination setup discussed in Section 2.2 was first employed to capture the PA images. The AcousticX data acquisition system software was synchronized to the laser acquisition mode. Two external triggers from the system were used for driving the laser. One trigger from the synch-1 port of the system went into the flash-lamp input port of the OPO laser and the other trigger from the synch-2 port fed the Q-Switch IN port of the laser, as shown in Figure 1b,c. After switching ON the 2-D B-mode RF data acquisition, the laser flash-lamp was first turned ON followed by (after 10s delay) the laser, from a PC connected to the laser. Once the laser was $\mathrm{ON}$ and synchronized with the data acquisition, PA data could be captured at a $10 \mathrm{~Hz}$ frame rate (limited by the PRF of the laser). Figure $2 \mathrm{~b}$ shows a PA image captured with the laser illumination at $850 \mathrm{~nm}$ wavelength and $40 \mathrm{~mJ} /$ pulse optical energy resulting in $<20 \mathrm{~mJ} / \mathrm{cm}^{2}$ optical fluence. As shown, all four pencil lead targets generated PA signals due to their higher optical absorptions compared to the intralipid medium.

To acquire the PA images with LED arrays, the laser arrangement was removed and the two $850 \mathrm{~nm}$ wavelength LED arrays were attached to the US transducer as shown in Figure 1e. The software of the Acoustic X system was set to the LED acquisition mode. The PRF for the LED arrays was selected as $4 \mathrm{KHz}$. Figure 2c shows a PA image captured using the LED array setup with a frame averaging of 128 . The achieved frame rate in this configuration was $30 \mathrm{~Hz}$ (128 averaging at $4 \mathrm{KHz}$ PRF). Further, to study the effect of frame averaging over the LED-PA imaging performance, the averaging was increased from 128 to $256,384,640,1280$ and 2560 frames, leading to frame rates of $15,10,6,3$, and $1.5 \mathrm{~Hz}$, respectively. The corresponding PA images are shown in Figure 2d-h.

In order to perform an effective comparison of the captured PA images with the laser and the LED array illuminations, the raw PA data were extracted and analyzed in a local computer using MATLAB software. First, frequency domain reconstruction [57] was performed to beamform the PA images from the raw data. All beamformed PA images were then log-compressed, maintaining the same $70 \mathrm{~dB}$ scale, as presented in Figure $2 \mathrm{~b}-\mathrm{h}$, for effective comparison. Further, to quantitatively compare these PA images, the SNR study was performed over all four pencil lead targets. For calculating the SNR, peak PA signal at the target locations (over a circular region surrounding the targets) and the mean noise adjacent to each target (over a similar circular region at same depth as targets) were calculated over the linear beamformed PA images. The calculated values of peak signal, mean noise and SNR for the four targets over the PA images corresponding to the varying frame averaging of laser acquisition $(1,2,4,10,128$ frame averages leading to $10 \mathrm{~Hz}$, $5 \mathrm{~Hz}, 2.5 \mathrm{~Hz}, 1 \mathrm{~Hz}$, and $78 \mathrm{mHz}$ frame rates when computed offline) and the LED array acquisition at varying frame rates are presented in Table 1 . The calculations at $78 \mathrm{mHz}$ with the laser system were specifically performed in order to compare the SNR of two systems at the same frame averaging-i.e., 128 frames (LED $30 \mathrm{~Hz}$ ). As shown, the peak PA signal for the laser acquisition is about two log orders higher than that for the LED arrays. However, the mean noise in the case of laser illumination is even higher (up to three log orders), leading to a lower SNR, especially when compared at shallow imaging depths. 
Plots in Figure 2i-l present the SNR trends observed with varying frame rates of LED array acquisitions for the four targets. The corresponding value of SNR for the laser acquisition at $10 \mathrm{~Hz}$ is also marked in each of these plots. For the targets lower than $30 \mathrm{~mm}$ depth, the LED-based PAI at $10 \mathrm{~Hz}$ frame rate continued to show a higher SNR. Target-4 at $34 \mathrm{~mm}$ depth was detected at a higher SNR with the laser illumination as compared to the LED arrays at the same frame rate. However, increasing the PA frame averaging further for the LED arrays acquisition led to a lower frame rate $(<10 \mathrm{~Hz})$, and helped in boosting the SNR value for higher depth targets. Further, when maintaining same frame averaging (128 frames) for the laser $(78 \mathrm{mHz}$ frame rate) and the LED arrays ( $30 \mathrm{~Hz}$ frame rate), significantly high SNRs were observed for all four targets with the laser, sacrificing the real-time imaging.

(a)
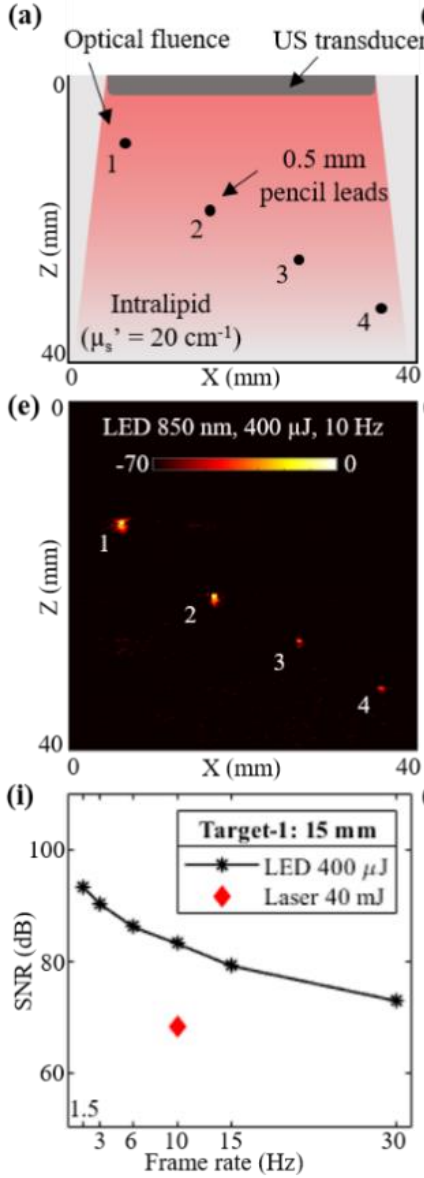

(b)
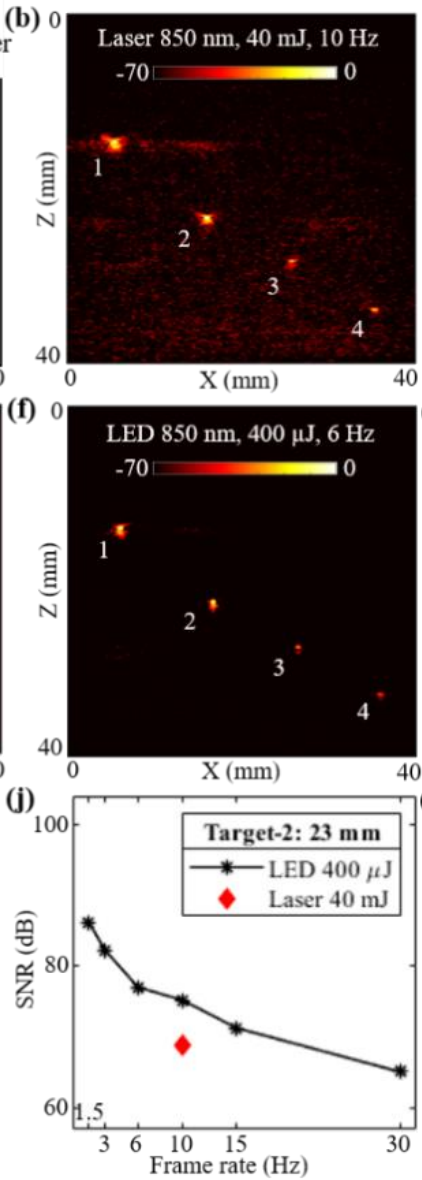
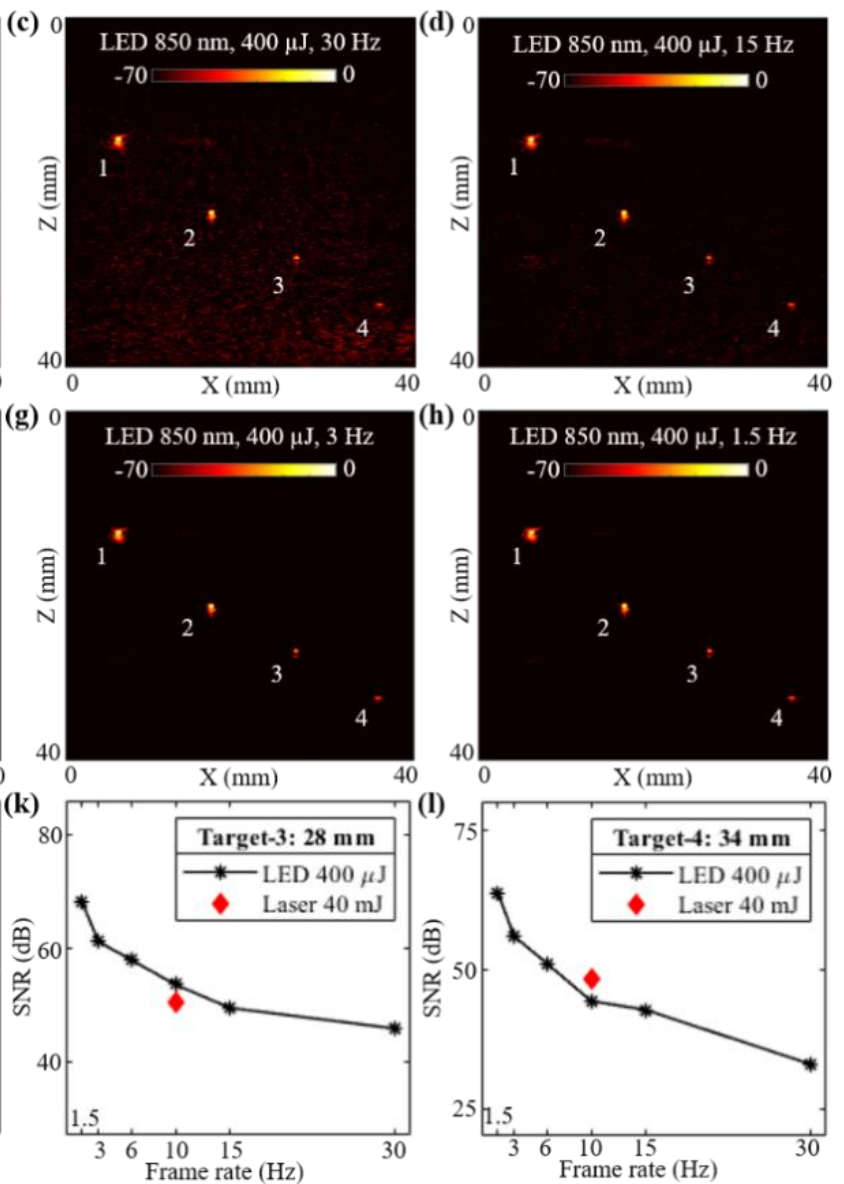

Figure 2. Performance evaluation of LED array-based and laser-illumination-based photoacoustic (PA) imaging in intralipid scattering phantom. (a) Shows the schematic of a scattering phantom with four $0.5 \mathrm{~mm}$ diameter pencil leads placed at 15, 23, 28 and $34 \mathrm{~mm}$ depth from the ultrasound (US) transducer inside intralipid medium mimicking an optical reduced scattering coefficient of $20 \mathrm{~cm}^{-1}$. (b) Shows the PA imaging results with $850 \mathrm{~nm}$ laser illumination at $10 \mathrm{~Hz}$ and $40 \mathrm{~mJ}$ optical energy with $<20 \mathrm{~mJ} / \mathrm{cm}^{2}$ optical fluence on the phantom surface. (c-h) Show the PA imaging results with LED array-based illumination at an $850 \mathrm{~nm}$ wavelength, a total of $400 \mu \mathrm{J}$ output energy from the two LED arrays and frame rates of 30,15, 10, 6,3 and $1.5 \mathrm{~Hz}$, respectively. (i-1) Show the plots of signal-to-noise ratio (SNR) with respect to the acquisition frame rates comparing LED array and laser-illumination-based PA imaging performance for the pencil lead targets at four depths. 
Table 1. Peak PA signal, average background noise and signal-to-noise ratio (SNR) values for four pencil lead targets located $15,23,28$ and $34 \mathrm{~mm}$ deep inside scattering phantom imaged with LED array and laser illuminations at varying frame rates.

\begin{tabular}{|c|c|c|c|c|c|c|c|c|c|c|c|c|}
\hline \multirow[b]{2}{*}{ Configuration } & \multicolumn{3}{|c|}{ Target-1: 15 mm } & \multicolumn{3}{|c|}{ Target-2: $23 \mathrm{~mm}$} & \multicolumn{3}{|c|}{ Target-3: $28 \mathrm{~mm}$} & \multicolumn{3}{|c|}{ Target-4: $34 \mathrm{~mm}$} \\
\hline & $\begin{array}{l}\text { Signal } \\
\text { (a.u.) }\end{array}$ & $\begin{array}{l}\text { Noise } \\
\text { (a.u.) }\end{array}$ & $\begin{array}{l}\text { SNR } \\
\text { (dB) }\end{array}$ & $\begin{array}{c}\text { Signal } \\
\text { (a.u.) }\end{array}$ & $\begin{array}{l}\text { Noise } \\
\text { (a.u.) }\end{array}$ & $\begin{array}{l}\text { SNR } \\
\text { (dB) }\end{array}$ & $\begin{array}{l}\text { Signal } \\
\text { (a.u.) }\end{array}$ & $\begin{array}{l}\text { Noise } \\
\text { (a.u.) }\end{array}$ & $\begin{array}{l}\text { SNR } \\
\text { (dB) }\end{array}$ & $\begin{array}{c}\text { Signal } \\
\text { (a.u.) }\end{array}$ & $\begin{array}{l}\text { Noise } \\
\text { (a.u.) }\end{array}$ & $\begin{array}{l}\text { SNR } \\
\text { (dB) }\end{array}$ \\
\hline Laser $10 \mathrm{~Hz}$ & 2.62E14 & $1.04 \mathrm{E} 11$ & 68.01 & $4.09 \mathrm{E} 14$ & $1.49 \mathrm{E} 11$ & 68.77 & $7.96 \mathrm{E} 13$ & $2.38 \mathrm{E} 11$ & 50.48 & $4.19 \mathrm{E} 13$ & $1.62 \mathrm{E} 11$ & 48.24 \\
\hline Laser $5 \mathrm{~Hz}$ & $2.60 \mathrm{E} 14$ & $5.01 \mathrm{E} 10$ & 74.31 & $4.25 \mathrm{E} 14$ & 7.39E10 & 75.18 & $8.58 \mathrm{E} 13$ & $1.43 \mathrm{E} 11$ & 55.55 & $4.38 \mathrm{E} 13$ & $8.81 \mathrm{E} 10$ & 53.93 \\
\hline Laser $2.5 \mathrm{~Hz}$ & $2.58 \mathrm{E} 14$ & $2.94 \mathrm{E} 10$ & 78.85 & 4.07E14 & $4.26 \mathrm{E} 10$ & 79.59 & 8.17E13 & $8.26 \mathrm{E} 10$ & 59.90 & $3.8 \mathrm{E} 13$ & $4.01 \mathrm{E} 10$ & 59.55 \\
\hline Laser $1 \mathrm{~Hz}$ & $2.58 \mathrm{E} 14$ & $1.44 \mathrm{E} 10$ & 85.05 & $4.05 \mathrm{E} 14$ & $2.25 \mathrm{E} 10$ & 85.10 & $7.89 \mathrm{E} 13$ & $6.64 \mathrm{E} 10$ & 61.50 & $3.79 \mathrm{E} 13$ & $1.66 \mathrm{E} 10$ & 67.18 \\
\hline Laser $78 \mathrm{mHz}$ & 2.47E14 & $5.66 \mathrm{E} 09$ & 92.79 & 3.39E14 & 7.95E09 & 92.58 & $5.94 \mathrm{E} 13$ & $3.86 \mathrm{E} 10$ & 63.80 & $2.89 \mathrm{E} 13$ & 3.35E09 & 78.50 \\
\hline LED $1.5 \mathrm{~Hz}$ & $3.62 \mathrm{E} 12$ & 8.06E07 & 93.06 & $1.95 \mathrm{E} 12$ & $9.59 \mathrm{E} 07$ & 86.17 & $2.06 \mathrm{E} 11$ & $8.16 \mathrm{E} 07$ & 68.04 & $8.59 \mathrm{E} 10$ & $5.70 \mathrm{E} 07$ & 63.55 \\
\hline LED $3.0 \mathrm{~Hz}$ & 3.57E12 & $1.11 \mathrm{E} 08$ & 90.14 & $1.94 \mathrm{E} 12$ & $1.50 \mathrm{E} 08$ & 82.33 & $1.84 \mathrm{E} 11$ & $1.62 \mathrm{E} 08$ & 61.13 & $7.80 \mathrm{E} 10$ & $1.27 \mathrm{E} 08$ & 55.79 \\
\hline LED $6.0 \mathrm{~Hz}$ & $3.69 \mathrm{E} 12$ & $1.84 \mathrm{E} 08$ & 86.01 & $1.89 \mathrm{E} 12$ & $2.65 \mathrm{E} 08$ & 77.04 & 2.14E11 & 2.72E08 & 57.90 & $8.39 \mathrm{E} 10$ & 2.39E08 & 50.92 \\
\hline LED $10 \mathrm{~Hz}$ & $3.66 \mathrm{E} 12$ & 2.57E08 & 83.06 & $2.06 \mathrm{E} 12$ & $3.55 \mathrm{E} 08$ & 75.26 & $1.95 \mathrm{E} 11$ & 4.08E08 & 53.58 & 7.64E10 & 4.70E08 & 44.22 \\
\hline LED $15 \mathrm{~Hz}$ & $3.56 \mathrm{E} 12$ & 3.98E08 & 79.03 & $1.86 \mathrm{E} 12$ & $5.06 \mathrm{E} 08$ & 71.31 & 2.07E11 & 6.98E08 & 49.45 & $8.36 \mathrm{E} 10$ & 6.18E08 & 42.62 \\
\hline LED $30 \mathrm{~Hz}$ & $3.70 \mathrm{E} 12$ & 8.49E08 & 72.79 & $1.95 \mathrm{E} 12$ & $1.08 \mathrm{E} 09$ & 65.12 & $2.38 \mathrm{E} 11$ & $1.21 \mathrm{E} 09$ & 45.84 & 7.22E10 & 1.63E09 & 32.92 \\
\hline
\end{tabular}

In order to validate the optical properties of our phantom, we calculated the $\mu_{\text {eff }}$ value from the experimental peak PA signal values for LED $10 \mathrm{~Hz}$ data. Using the Beer Lambert's principle, i.e., $I(z)=I_{o} e^{-\mu_{e f f} z}$, where $I(z)$ is the PA intensity at depth $(\mathrm{z}) \mathrm{cm}$ and $I_{o}$ is the intensity at zero depth, and taking the ratio of two equations at two different depths $\left(z_{1}\right.$ at $1.5 \mathrm{~cm}$ and $z_{2}$ at $\left.3.4 \mathrm{~cm}\right)$ will cancel out the $I_{o}$ and lead to the $\mu_{\text {eff }} 2.034 \mathrm{~cm}^{-1}$. This closely matches to the previously reported values in the range of 1.734 to $2.456 \mathrm{~cm}^{-1}$, mentioned above in the phantom description.

\subsection{Photoacoustic Imaging Comparision of LED Arrays and Laser Illuminations over Chicken Tissue Phantom}

To compare the deep tissue PAI capabilities of the low-power LED arrays and the highpower laser illuminations, a multilayer chicken tissue phantom was designed. Figure $3 \mathrm{a}$ shows the schematic of the chicken tissue phantom with five layers of chicken breast tissue stacked inside a water tank, with an estimated optical absorption and reduced scattering coefficients of 0.1 to $0.2 \mathrm{~cm}^{-1}$ and 1.0 to $5 \mathrm{~cm}^{-1}$, respectively, with an $850 \mathrm{~nm}$ wavelength [58], leading to an effective attenuation coefficient $\left(\mu_{\text {eff }}\right)$ in the range of 0.575 to $1.766 \mathrm{~cm}^{-1}\left(\mu_{e f f}=\sqrt{3 \mu_{a}\left(\mu_{a}+\mu_{s}^{\prime}\right)}\right)$. Four pencil leads with diameters of $0.5 \mathrm{~mm}$ were placed in between the chicken tissue layers as shown. The measured depths of these pencil lead targets from the top layer of the chicken tissue were 11, 18, 24, and $31 \mathrm{~mm}$, respectively.

With the above-described phantom, a laser-illumination setup was used to acquire the US and PA images at $10 \mathrm{~Hz}$, maintaining the same $40 \mathrm{~mJ}$ output energy $\left(<20 \mathrm{~mJ} / \mathrm{cm}^{2}\right.$ optical fluence on the phantom surface) and laser with an $850 \mathrm{~nm}$ wavelength. The captured raw data from the AcousticX software were reconstructed in MATLAB to further perform the quantitative comparison. Figure $3 b$ shows the beamformed B-mode US image of the chicken tissue phantom, at a $\log$ scale of $70 \mathrm{~dB}$, clearly highlighting the chicken tissue structure. The US image also shows the distance of the four pencil lead targets from the top layer of chicken tissue. Similarly, the PA raw data captured from the software were reconstructed, log-compressed and was overlaid on the US image to generate a coregistered US + PA image of the phantom. Figure $3 c$ shows the coregistered US and PA image at a $60 \mathrm{~dB}$ scale in order to keep the noise floor at the threshold. The beamformed PA image with $70 \mathrm{~dB} \log$ scale is shown in Figure $3 \mathrm{~d}$.

Without disturbing the phantom, the PA images were subsequently acquired with the LED arrays setup. The laser arrangement was removed and the two $850 \mathrm{~nm}$ LED arrays were attached to the US probe. With the $4 \mathrm{KHz}$ PRF of the LED arrays, the PA images were captured at varying frame averaging settings, similar to the settings used for Section 3.1, leading to the frame rates of 30,15, 10, 6, 3 and $1.5 \mathrm{~Hz}$. The captured raw data from the AcousticX software were again extracted in the MATLAB software and reconstructed to generate B-mode PA images for comparing with the laser PA images. All PA images were compressed at the $70 \mathrm{~dB} \log$ scale for effective comparison. Four representative PA images at 30, 15, 10, and $6 \mathrm{~Hz}$, respectively, are shown in Figure 3e-h. 

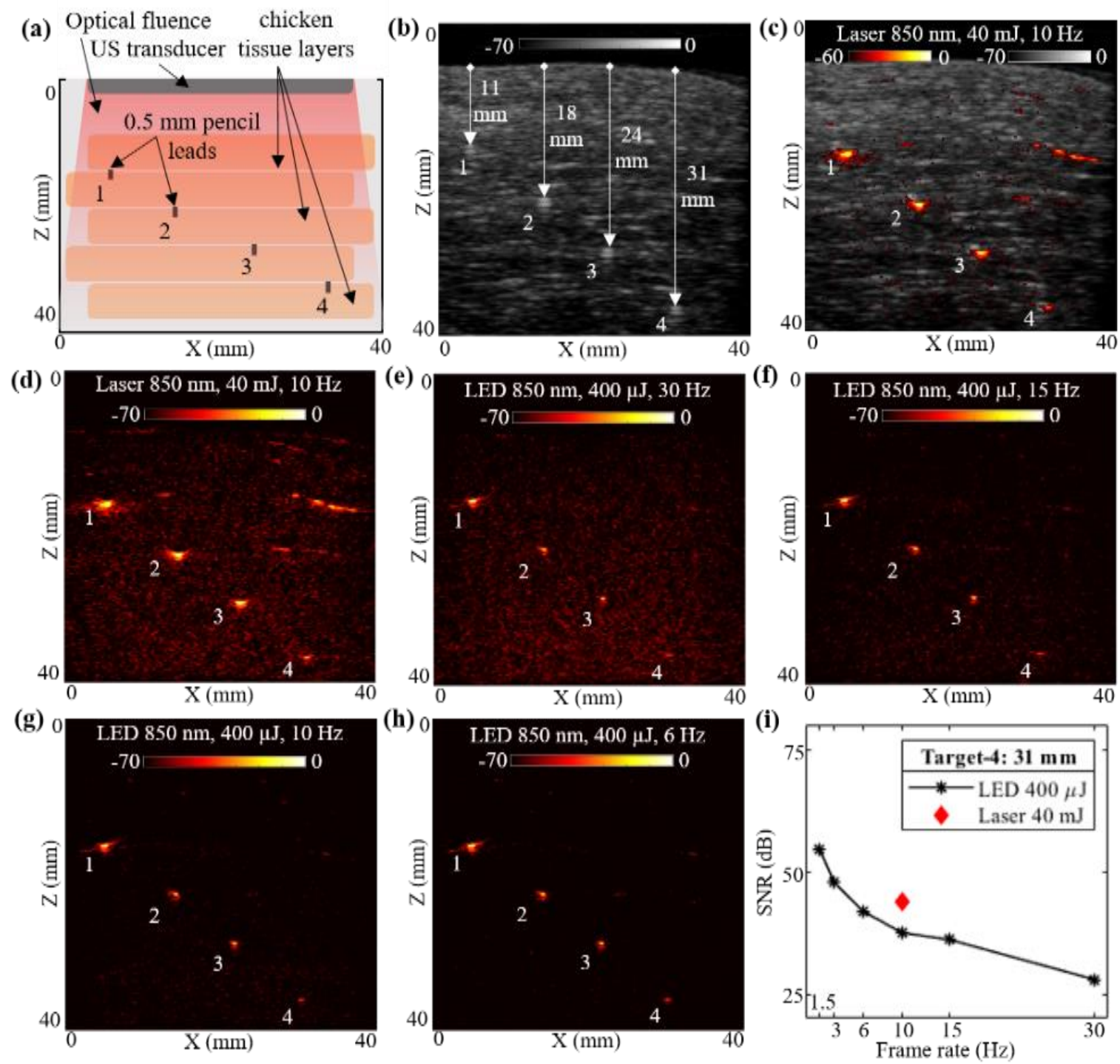

Figure 3. Performance evaluation of LED array-based and laser-based photoacoustic (PA) imaging in chicken tissue phantom. (a) Shows a schematic of chicken tissue phantom with five layers of chicken breast tissue stacked inside water tank. The positions of four $0.5 \mathrm{~mm}$ diameter pencil lead targets placed in between the chicken tissue layers are also shown. (b-d) Show the B-mode ultrasound (US), coregistered US + PA and PA image, respectively, captured with laser-based illumination at $850 \mathrm{~nm}$ wavelength and $10 \mathrm{~Hz}$ high pulse repetition frequency (PRF). (e-h) Show the PA imaging results obtained by imaging the chicken tissue phantom using LED array-based illumination, at $850 \mathrm{~nm}$ illumination and frame rates of 30,15,10, and $6 \mathrm{~Hz}$, respectively. (i) Shows the plot of signal-to-noise ratio (SNR) with respect to the frame rate comparing the LED array and laser-illumination-based PA imaging performance for the deepest pencil lead target (target-4).

Further, for a quantitative comparison of high-power laser versus low power LED array-based acquisitions over the chicken tissue phantom, the SNR study was performed over the linear beamformed images in MATLAB. For this experiment, we studied the SNR of the shallowest target (target-1 located $11 \mathrm{~mm}$ ) and the deepest target (target-4 located $31 \mathrm{~mm}$ ) deep inside chicken breast tissue. For calculating the SNR, the peak signal at target locations and the mean noise adjacent to these targets were calculated. The values of peak PA signal, mean adjacent noise and the SNR for laser-based and LED array-based acquisitions at varying frame rates are listed in Table 2 . The peak signal as well as mean noise for laser acquisition at $10 \mathrm{~Hz}$ is about two to three log orders of magnitude higher compared to the LED arrays. However, the SNR for LEDs is still comparable to the lasers. Figure $3 \mathrm{i}$ also demonstrates the trend of SNR for target-4, comparing the laser and the LED array illuminations at varying frame rates. For a $31 \mathrm{~mm}$ deep target, LED arrays provide 
close to $37.51 \mathrm{~dB}$ SNRs, whereas laser illumination provides about $43.75 \mathrm{~dB}$ SNR at a $10 \mathrm{~Hz}$ frame rate. When increasing the frame averaging, hence a reduction in the frame rate, LED arrays show significant improvement of SNR for the same target, up to $54.47 \mathrm{~dB}$ at $1.5 \mathrm{~Hz}$, which is higher than the SNR of laser illumination at $10 \mathrm{~Hz}$. These results demonstrate the capabilities of LED arrays to image deeper inside realistic tissue medium and motivated us to further study how they compare with high-power laser sources for imaging in vivo human vasculature.

Table 2. Peak PA signal, average background noise and signal-to-noise ratio (SNR) values for pencil lead targets located 11 and $31 \mathrm{~mm}$ deep inside chicken breast tissue imaged with LED array and laser illuminations at varying frame rates.

\begin{tabular}{lcccccc}
\hline & \multicolumn{3}{c}{ Target-1: 11 mm } & \multicolumn{3}{c}{ Target-4: 31 mm } \\
\cline { 2 - 7 } Configuration & $\begin{array}{c}\text { Signal } \\
\text { (a.u.) }\end{array}$ & $\begin{array}{c}\text { Noise } \\
\text { (a.u.) }\end{array}$ & $\begin{array}{c}\text { SNR } \\
\text { (dB) }\end{array}$ & $\begin{array}{c}\text { Signal } \\
\text { (a.u.) }\end{array}$ & $\begin{array}{c}\text { Noise } \\
\text { (a.u.) }\end{array}$ & $\begin{array}{c}\text { SNR } \\
\text { (dB) }\end{array}$ \\
\hline Laser 10 Hz & $1.4 \mathrm{E} 14$ & $1.2 \mathrm{E} 11$ & 61.63 & $2.1 \mathrm{E} 12$ & $1.4 \mathrm{E} 11$ & 43.75 \\
LED 1.5 Hz & $2.1 \mathrm{E} 12$ & $6.8 \mathrm{E} 07$ & 89.71 & $3.5 \mathrm{E} 10$ & $6.6 \mathrm{E} 07$ & 54.47 \\
LED 3.0 Hz & $2.1 \mathrm{E} 12$ & $9.2 \mathrm{E} 08$ & 87.35 & $3.4 \mathrm{E} 10$ & $1.4 \mathrm{E} 08$ & 47.76 \\
LED 6.0 Hz & $2.1 \mathrm{E} 12$ & $1.6 \mathrm{E} 08$ & 82.47 & $2.8 \mathrm{E} 10$ & $2.2 \mathrm{E} 08$ & 41.89 \\
LED 10 Hz & $2.0 \mathrm{E} 12$ & $2.8 \mathrm{E} 08$ & 77.13 & $3.2 \mathrm{E} 10$ & $4.3 \mathrm{E} 08$ & 37.51 \\
LED 15 Hz & $2.1 \mathrm{E} 12$ & $3.7 \mathrm{E} 08$ & 75.03 & $3.6 \mathrm{E} 10$ & $5.7 \mathrm{E} 08$ & 36.08 \\
LED 30 Hz & $2.1 \mathrm{E} 12$ & $7.4 \mathrm{E} 08$ & 69.06 & $2.9 \mathrm{E} 10$ & $1.2 \mathrm{E} 09$ & 27.98 \\
\hline
\end{tabular}

Similar to Section 3.1, we validated the optical properties of this chicken tissue phantom by calculating the $\mu_{e f f}$ value from the experimental peak PA signal values for LED $10 \mathrm{~Hz}$ data. The experimentally calculated $\mu_{\text {eff }}\left(2.067 \mathrm{~cm}^{-1}\right)$ is slightly higher than the previously calculated range mentioned above in the phantom description. The small discrepancy could be due to chicken tissue heterogeneity-prolonged imaging of chicken tissue in water medium that increases the optical attenuation.

\subsection{Photoacoustic Imaging Comparison of LED Arrays and Laser Sources: Resolution Study}

In this subsection, the spatial resolutions of the two optical illumination setups, the LED-PAUS and the laser-PAUS, are characterized. A $30 \mu \mathrm{m}$ carbon fiber was placed in a bath containing water mixed with intralipid to obtain a scattering medium of $3 \mathrm{~cm}^{-1}$. First, the two $850 \mathrm{~nm}$ LED arrays were attached to the US probe and a B-mode PA image of the phantom was acquired. The raw data captured were extracted in the MATLAB software and reconstructed to generate a B-mode image. The log-compressed B-mode PA image at a $30 \mathrm{~dB}$ scale is shown in Figure $4 \mathrm{a}$. Figure $4 \mathrm{~b}$ presents a sample zoomed time trace of an A-line across the target region for the PA data acquired with LED array illumination. To calculate the spatial resolution for this carbon fiber target, the line-spread functions of the PA amplitudes are plotted in the lateral and axial directions, respectively, as shown in Figure $4 \mathrm{c}, \mathrm{d}$. The obtained lateral and axial resolutions with a full-width-half-maximum (FWHM) approach are 350 and $210 \mu \mathrm{m}$, respectively.

To acquire the PA data with laser-illumination, the LED arrays were removed from the US probe and the laser fiber setup was attached without disturbing the phantom. The captured PA data were extracted and beamformed. Figure $4 \mathrm{e}$ shows the B-mode PA image, at a $30 \mathrm{~dB}$ scale, obtained for the same carbon fiber phantom using laser-illumination at $850 \mathrm{~nm}$ with $40 \mathrm{~mJ}$ output energy and a $10 \mathrm{~Hz}$ frame rate. A sample zoomed time trace of an A-line across the target region for the PA data acquired with the laser-illumination is shown in Figure $4 \mathrm{f}$. The line-spread functions of the PA amplitudes in the lateral and axial directions with the laser illumination are shown in Figure $4 \mathrm{~g}$,h. The obtained lateral and axial resolutions using an FWHM approach for the laser illumination are 355 and $203 \mu \mathrm{m}$, respectively. 

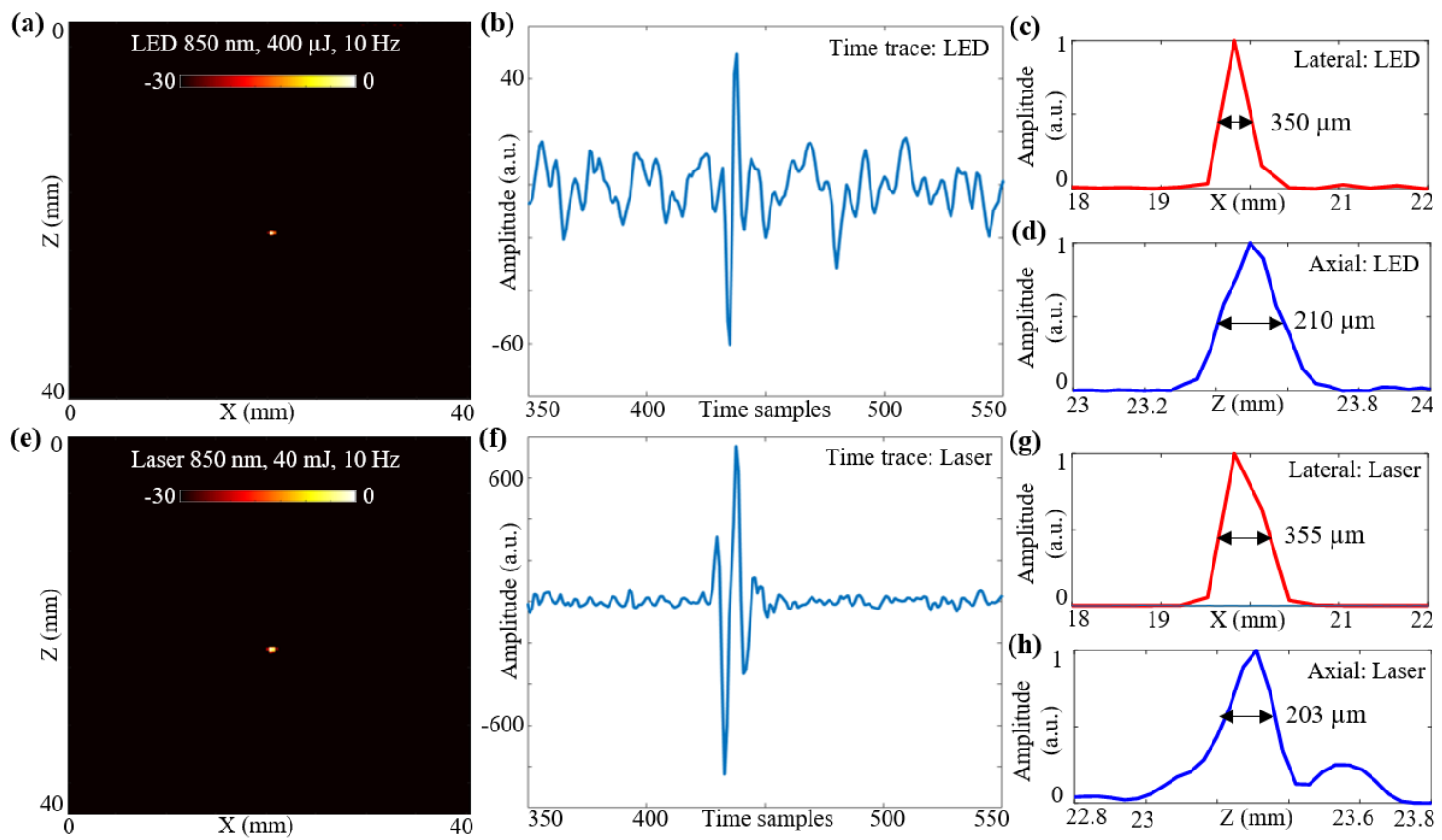

Figure 4. Resolution study for LED array-based and laser-illumination-based photoacoustic (PA) imaging. (a) B-mode PA image obtained for a $30 \mu \mathrm{m}$ carbon fiber placed in an intralipid-based phantom using LED illumination at $850 \mathrm{~nm}$ with a frame rate of $10 \mathrm{~Hz}$. (b) Shows zoomed time trace of an A-line across the target region for the PA data acquired with LED illumination. (c,d) Show the line-spread functions of the PA amplitudes for the carbon fiber target plotted in the lateral and axial directions, respectively, when imaged with LED setup. The obtained lateral and axial resolutions with the full-width-half-maximum (FWHM) approach are 350 and $210 \mu \mathrm{m}$, respectively. (e) B-mode PA image obtained for same carbon fiber phantom using laser illumination at $850 \mathrm{~nm}$ and $10 \mathrm{~Hz}$ frame rate. (f) Shows the time trace for the PA data acquired with laser illumination. (g,h) Show the line-spread functions of the PA amplitudes in lateral and axial directions with the laser illumination. The obtained lateral and axial resolutions using the FWHM approach are 355 and $203 \mu \mathrm{m}$, respectively.

\subsection{In Vivo Photoacoustic Imaging Comparison of LED Arrays and Laser Illumination over} In Vivo Human Wrist

In this subsection, the photoacoustic vascular imaging capabilities of the LED array and the laser illumination were compared by in vivo imaging of a healthy human volunteer's wrist vasculature. The volunteer was a healthy 25-year-old European male, and the experiment was conducted by following the internal imaging protocol of CYBERDYNE, INC (Rotterdam, The Netherlands) for healthy-volunteer imaging studies. For this study, the volunteer's right hand was positioned inside a large water tank, as shown in Figure 5a. The probe was positioned such that one of the major blood vessels, which supplies blood to the forearm and hand, is within the field-of-view (FOV).

The hand was first imaged with the laser-PAUS setup by attaching the laser fiber holders to the US probe, as shown in Figure 5a. The laser was operated at $850 \mathrm{~nm}$ wavelength, $10 \mathrm{~Hz}$ PRF, and delivered output optical energy of $40 \mathrm{~mJ}$. This allowed ANSI safety limits of $<20 \mathrm{~mJ} / \mathrm{cm}^{2}$ optical fluence on the hand surface [59]. During the real-time data acquisition, the probe was aligned such that the major blood vessel could be seen running parallel to the skin surface in the PA images. The captured US and PA raw data using the AcousticX software were later extracted in the MATLAB software and were reconstructed to generate the beamformed images. The beamformed, log-compressed B-mode US, PA and the coregistered US + PA images are shown in Figure $5 b-d$. The US image showed the anatomical features along the depth of human wrist, whereas the PA image highlighted the major blood vasculature. There was fairly strong correspondence between the locations of the blood vessel in the PA image and the appearance of anechoic regions in the US image. 
Based on the anatomy of the vasculature in human wrist, the PA signals $\sim 5 \mathrm{~mm}$ below the skin surface may have corresponded to the radial artery that travels across the front of the elbow, deep under the muscle until it comes to the wrist where it comes close to the skin surface. This is also marked with a white arrow in the PA image in Figure 5c.

To compare these laser-illumination results of the human wrist with the LED arrays, the laser-fiber attachments were gently removed without disturbing the location of the US probe. The two $850 \mathrm{~nm}$ LED arrays were then attached to the US probe, as shown in Figure 5e. With a PRF of $4 \mathrm{KHz}$ and frame averaging of 384, leading to a frame rate of $10 \mathrm{~Hz}$, the US and PA frames were captured using the LED array setup. The US and PA raw data were then reconstructed in MATLAB. Figure $5 \mathrm{f}-\mathrm{h}$ show the beamformed log-compressed B-mode US, PA and coregistered US + PA images for the human wrist. As in the case of laser illumination, the LED array-based PA images also imaged the same vasculature below the skin surface. The radial artery present $\sim 5 \mathrm{~mm}$ below the skin was clearly visible with the LED array-based acquisition as well.
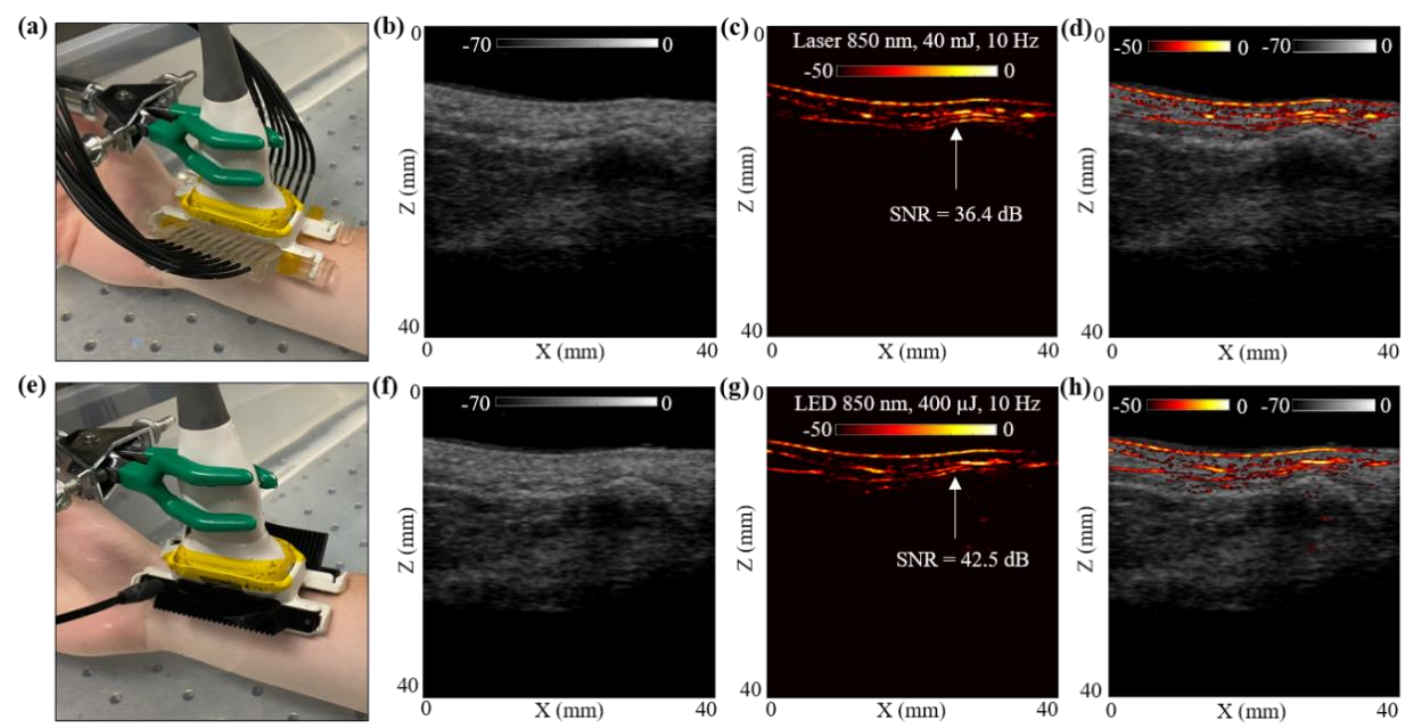

Figure 5. In vivo comparison of LED array-based and laser-based PA vasculature imaging over the right-hand wrist of a healthy 25-year-old male human volunteer. (a) Shows the experimental setup with the right-hand wrist placed inside a big water bath for the laser-based PA imaging. (b-d) Show the obtained US, PA and coregistered US + PA images for the setup shown in (a). (e) Shows the setup with LED arrays. (f-h) Show the obtained US, PA, and coregistered US + PA images for the setup shown in (e).

To further compare the two setups quantitatively, an SNR comparison study was performed for the radial artery, as marked with white arrows in Figure $5 \mathrm{c}, \mathrm{g}$. To calculate the SNR, the peak PA signal at the artery and the mean noise adjacent to the artery region was calculated over the linear beamformed PA images resulting from the laser-illumination and the LED array-based acquisitions. Table 3 presents the values of peak PA signal, mean noise and the SNR. The SNR values are also marked in the Figure 5c,g. Both the peak signal and mean noise with the laser are up to three log orders of magnitude higher compared to the LEDs. However, the SNR value for the laser-illumination-based PA image was about $6 \mathrm{~dB}$ lower than the SNR with the LED array acquisition. This follows the trend observed for the controlled tissue phantom studies discussed in Section 3.1, where the shallow targets $(<30 \mathrm{~mm})$ inside an intralipid medium were detected with higher SNRs using LED arrays compared to the laser illumination, maintaining the same frame rate. 
Table 3. Peak PA signal, average background noise and signal-to-noise ratio (SNR) values for blood vessel target located $\sim 5 \mathrm{~mm}$ below the skin surface of right-hand wrist of a healthy 25 -year-old male human volunteer imaged with LED array and laser illumination at $10 \mathrm{~Hz}$ frame rate.

\begin{tabular}{cccc}
\hline \multirow{2}{*}{ Configuration } & \multicolumn{3}{c}{ Target: Blood Vessel $\sim \mathbf{5 m}$ below Skin Surface of Human Wrist } \\
\cline { 2 - 4 } & Signal (a.u.) & Noise (a.u.) & SNR (dB) \\
\hline Laser $\mathbf{1 0 ~} \mathbf{~ H z}$ & $3.7 \mathrm{E} 13$ & $5.7 \mathrm{E} 11$ & 36.37 \\
LED $\mathbf{1 0 ~} \mathbf{~ z z}$ & $5.3 \mathrm{E} 10$ & $4.0 \mathrm{E} 08$ & 42.49 \\
\hline
\end{tabular}

3.5. In Vivo Photoacoustic Imaging Comparison of LED Arrays and Laser Illumination: In Vivo Human Forearm

This subsection presents in vivo 3-D mapping of the vasculature inside a human volunteer's forearm, using the LED array-based and the laser-based illuminations. For this study, the volunteer was a healthy 25-year-old European male, and the experiment was conducted by following the internal imaging protocol of CYBERDYNE, INC (Rotterdam, The Netherlands) for healthy-volunteer imaging experiments. The forearm of the volunteer was submerged in water. The US probe was fixed on a linear translation stage, translating in the Y-direction, as shown in Figure 6a.

For the laser acquisition, the laser-illumination setup was attached to the US probe. After switching ON the laser software, the RF data acquisition on the AcousticX software were first turned ON. Note that the 3-D data acquisition feature of the AcousticX software does not work for the laser mode. Therefore, to capture the data corresponding to the Y-direction scan, a manual stage motion feature was used while acquiring the data in the 2-D RF acquisition mode. The linear stage was translated for $60 \mathrm{~mm}$ in the Y-direction while capturing the RF data. The laser was tuned to $850 \mathrm{~nm}$ at $40 \mathrm{~mJ}$ output energy $\left(<20 \mathrm{~mJ} / \mathrm{cm}^{2}\right.$ optical fluence on the hand surface) and $10 \mathrm{~Hz}$ PRF. The captured PA raw data were later extracted and reconstructed in MATLAB. Initial PA frames captured prior to the stage motion were discarded for the $3-\mathrm{D}$ volumetric reconstruction. Figure $6 \mathrm{~b}$ presents the maximum-intensity-projection (MIP) of the 3-D PA volume for full depth, highlighting the major vasculature present in the human forearm. To better visualize the vasculature deeper than $\sim 5 \mathrm{~mm}$, a deep tissue MIP of the PA volume is also shown in Figure 6c. Considering the initial $15 \mathrm{~mm}$ stand-off of the US probe from the human skin surface, the deep tissue MIP fell within $Z=20$ to $40 \mathrm{~mm}$, where $Z$ is the depth dimension. Since deeper blood vessels show weak PA intensities, the MIP image in Figure $6 \mathrm{c}$ was scaled to $30 \mathrm{~dB}$ as opposed to the full depth MIP in Figure 6b, which was scaled to $50 \mathrm{~dB}$.

After the laser acquisition, the stage was manually moved back to the home position and the laser setup was detached from the US probe without disturbing the position of the forearm with respect to the probe. To acquire the LED array-based PA 3-D scan data, two $850 \mathrm{~nm}$ LED arrays were attached to the US probe. In this case, the AcousticX software directly allows 3-D PA data acquisition using the automatic 3-D scan feature. The same Ydirection translation stage was automatically scanned for $60 \mathrm{~mm}$ and the raw data captured were extracted in MATLAB. The beamformed PA frames were stitched in the Y-dimension to reconstruct a 3-D PA volume. Figure 6d,e present the full depth MIP and the deep tissue MIP of the 3-D PA volume, respectively.

Qualitatively, Figure 6b,e look very similar, indicating that both laser and LED arraybased illumination are able to map the major vasculature present inside human forearm. Minor differences in the SNR for deep vessels can be noticed when comparing the deep tissue MIPs presented in Figure 6c,f. For example, a deeper blood vessel running parallel to the $Y$-axis at around $X=20 \mathrm{~mm}$ (in Figure $6 \mathrm{c}, \mathrm{f}$ ) was detected with a better SNR with the laser source as compared to the LED arrays. Similarly, the deep tissue MIP from laser acquisition show other minor PA signals (at coordinates $X, Y=30 \mathrm{~mm}, 50 \mathrm{~mm}$ ), that are relatively weaker in the deep tissue MIP from the LED acquisitions, emphasizing the need for a high-power laser when imaging very deep $(>2 \mathrm{~cm})$ vasculature. 

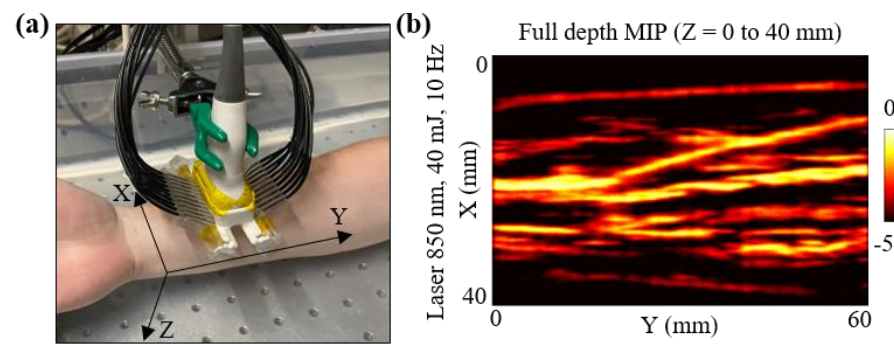

(d)

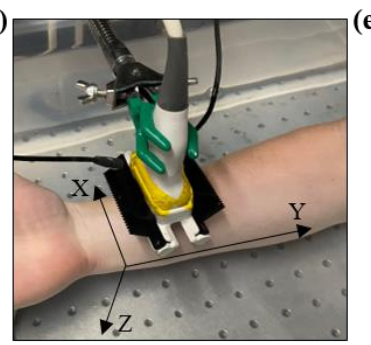

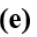

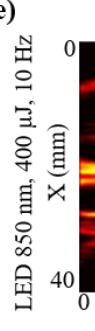

Full depth MIP ( $Z=0$ to $40 \mathrm{~mm}$ )

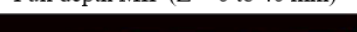

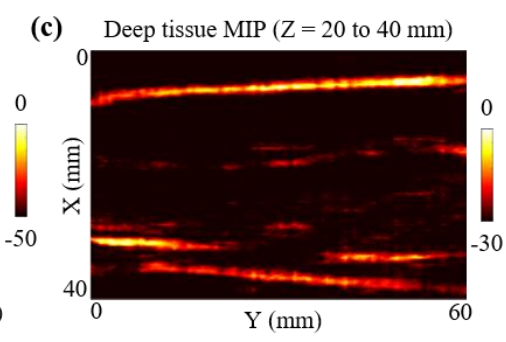

(f) Deep tissue $\operatorname{MIP}(\mathrm{Z}=20$ to $40 \mathrm{~mm})$

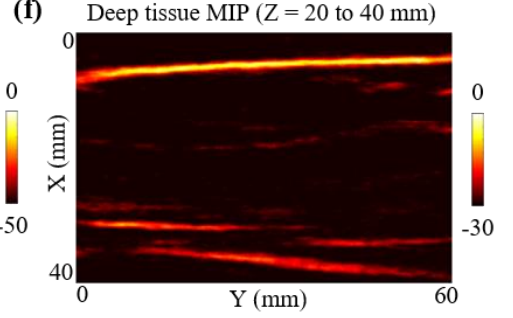

Figure 6. In vivo comparison of LED array-based and laser-based PA vasculature imaging over forearm of a healthy 25-year-old male human volunteer. (a) Shows the experimental setup with the right forearm placed inside big water bath for the laser-based PA imaging. (b,c) Show the obtained full depth and deep tissue maximum-intensity-projections (MIPs) of the reconstructed 3-D PA volume for the setup shown in (a). (d) Shows the setup with LED arrays. (e,f) Show the obtained full depth and deep tissue MIPs of the reconstructed 3-D PA volume for the setup shown in (d).

\section{Discussion}

To successfully translate PAI to the POC applications and to resource-limited settings, the cost and the overall size of the PAI systems need to be significantly cut down. LED arrays are one of the highly explored optical sources in the PA literature that are portable and significantly lower in cost and footprint. This study was focused on comparing the capabilities of these LED arrays (approximately USD 15K, including driver electronics) with a state-of-the-art high-power OPO laser (approximately USD 100K) for PAI. A commercial LED-PAUS system, AcousticX, was first adapted to perform sequential acquisitions of LED-PAUS and laser-PAUS. This ensured that the PA and US signals were detected by the same ultrasound transducer array and processed by the same data acquisition system. To further draw a fair comparison, the experimental setup shown in Figure 1 allowed for (1) similar optical illumination on the tissue surface in terms of the geometry, angle and aperture of illumination, and (2) a convenient, uninterrupted sequential acquisition of PA images with the two sources. To achieve uniform laser illumination on the tissue surface, two glass diffusers were attached to the output end of the laser fiber holders. For all the experimental studies presented in this work, the output optical energy for the laser and the LED arrays were maintained at $40 \mathrm{~mJ}$ and $400 \mu \mathrm{J} /$ pulse, respectively, with an $850 \mathrm{~nm}$ wavelength of illumination. A constant PRF of $10 \mathrm{~Hz}$ for the laser and $4 \mathrm{KHz}$ for the LED arrays were used for all studies. With no frame averaging, the obtained PA frame rate for laser was $10 \mathrm{~Hz}$. For the LED arrays, the PA frame averaging was varied from 128 to $256,384,640,1280$, and 2560 , achieving the frame rates of $30,15,10,6,3$, and $1.5 \mathrm{~Hz}$, respectively.

The first comparison study presented in Section 3.1 consisted of controlled experiments on an intralipid phantom shown in Figure 2. The four $0.5 \mathrm{~mm}$ pencil lead targets arranged diagonally along the depth of the scattering phantom were imaged using both laser and LED arrays. Detailed analysis of the SNR presented in Table 1 and plotted in Figure 2i-1 led to the following conclusions on the quantitative performance of the two sources. (1) At similar frame rates $(10 \mathrm{~Hz})$, LED-PAUS imaging showed higher SNR compared to laserPAUS imaging for targets up to an imaging depth of $30 \mathrm{~mm}$. (2) Laser-PAUS imaging provided a better SNR for the deeper targets $(>30 \mathrm{~mm})$ as compared to the LED-PAUS at $10 \mathrm{~Hz}$. (3) With increased frame averaging and hence a reduced overall frame rate $(<10 \mathrm{~Hz})$, 
the deep tissue $(>30 \mathrm{~mm}$ ) performance of LED arrays can be improved. For example, the target-4 located $34 \mathrm{~mm}$ deep was imaged with a $48.24 \mathrm{~dB}$ SNR using laser illumination and with $44.22 \mathrm{~dB}$ SNR using LED arrays, both at $10 \mathrm{~Hz}$. However, with increase in PA averaging (lower frame rates), LED arrays were able to provide an improved SNR of up to $50.92,55.79$, and $63.55 \mathrm{~dB}$, when imaged at 6,3 , and $1.5 \mathrm{~Hz}$ frame rates, respectively. Further, comparison of the magnitudes of peak PA signals from the target and mean noise surrounding the target for the two optical illuminations led to the following observations. While higher optical energy from the laser source generates a PA signal two to three log orders of magnitude higher compared to the lower-power LED arrays, the average noise floor from the background regions was also observed to be up to three to four log orders higher for the laser sources compared to LED arrays. In conclusion, the low-power LED arrays were effective due to lower noise floor that comes from the higher frame averaging possible with high PRF (4 KHz) of LED arrays.

Our next comparison study, presented in Section 3.2, involved a multilayer chicken tissue phantom with four $0.5 \mathrm{~mm}$ pencil lead targets embedded in between the five layers of chicken breast tissue. Figure 3 presented the qualitative comparison of laser-PAUS and LED-PAUS, whereas Table 2 highlighted the quantitative comparison of PAI performance for the deepest pencil lead target-i.e., target- 4 at $31 \mathrm{~mm}$. This study results further confirmed our observations with the intralipid scattering phantom presented in Section 3.1. For example: (1) both peak PA signal and mean noise from the laser illumination were up to three log orders of magnitude higher compared to the LED array illumination. (2) For the target- 4 at $31 \mathrm{~mm}$, the laser showed a $\sim 6 \mathrm{~dB}$ higher SNR compared to the LED arrays at a $10 \mathrm{~Hz}$ PA frame rate. (3) With the increase in the average, and hence reduced, frame rate $(<10 \mathrm{~Hz})$, LED arrays show higher SNR values than laser illumination, obtaining SNRs of 54.47 and $47.76 \mathrm{~dB}$ at 1.5 and $3 \mathrm{~Hz}$, respectively, for the same target- 4 as compared to an SNR of $43.75 \mathrm{~dB}$ with the laser.

The study presented in Section 3.3 compared the lateral and axial resolutions of the two setups, LED-PAUS and laser-PAUS, by imaging carbon fiber with a $30 \mu \mathrm{m}$ diameter inside an intralipid-based phantom. Although the pulse widths for the laser (5-7 ns) and the LED arrays (70 ns) in this study were not similar, the spatial resolution were shown to be in the same ranges-i.e., $\sim 350 \mu \mathrm{m}$ lateral and $\sim 205 \mu \mathrm{m}$ axial. This confirmed the fact that the spatial resolution here is mainly limited by the acoustic detection.

In the final study, in Section 3.4 we presented an in vivo LED-PAUS and laser-PAUS imaging of the wrist of a healthy human volunteer. Figure 5 presents the qualitative and quantitative comparison results for a radial artery seen $\sim 5 \mathrm{~mm}$ below the wrist skin surface. Analysis of the peak PA signal, mean noise and SNR for this radial artery is presented in Table 3. As observed with the studies presented in previous sections, for a shallow depth target, the LED-PAUS showed better SNR than the laser-PAUS at the same $10 \mathrm{~Hz}$ frame rates. The $\sim 5 \mathrm{~mm}$ deep radial artery was imaged with a $36.37 \mathrm{~dB}$ SNR with the laser compared to a $42.49 \mathrm{~dB}$ SNR with LED arrays. This study substantiates that the LED-PAUS imaging is an attractive choice for several preclinical and clinical applications.

Further, in Section 3.5, to test the capabilities of deep tissue in vivo vascular imaging, we scanned the right-hand forearm of a healthy human volunteer. The 3-D vasculature map, presented in Figure 6, demonstrated that the LED arrays could image all the vessels that could be seen with a laser source, especially at shallow depths of up to $1.5 \mathrm{~cm}$. For deeper $(>30 \mathrm{~mm})$ vessels, laser sources provided better signals. However, the amount of noise observed with the laser at those depths was also significantly high and thus limited the imaging performance when viewing deeper vessels. With an increased frame averaging with the LED arrays, the SNR of deeper vessels can potentially be improved.

The conclusive observations made by studying the performance of the two optical sources bring us to the following remarks. (1) LED arrays exhibit a strong potential for translating PAI systems to the resource-limited settings. (2) Higher frame averaging enhances the LED-PAUS imaging performance, especially for deeper targets $(>30 \mathrm{~mm})$, making it suitable for deep tissue imaging; however, this sacrifices the frame rate. To further improve the performance 
of LED-PAUS imaging, the state-of-the-art machine learning approaches can be employed that can help in (1) further boosting the frame rates by avoiding the need for higher frame averaging [60,61], and (2) improving the SNR for deep tissue targets [61,62].

\section{Conclusions}

Photoacoustic imaging capabilities of low-cost and low-power LED arrays were compared head-to-head with a high-power laser source using both tissue-mimicking phantoms and in vivo human subjects. The experimental observations on different tissue mimicking phantom studies demonstrated (1) high PRFs of LED arrays can be leveraged for averaging PA frames to achieve high SNR up to an imaging depth of $30 \mathrm{~mm}$ with $10 \mathrm{~Hz}$ frame rate, (2) high-power laser sources show higher SNRs for deeper targets $(>30 \mathrm{~mm})$ compared to the LED arrays at $10 \mathrm{~Hz}$ and (3) with increased frame averaging $(<10 \mathrm{~Hz})$, the SNR of a target at $34 \mathrm{~mm}$ depth with LED-PAUS closely matched that of laser-PAUS. The in vivo human hand vasculature imaging studies presented in this work also demonstrated similar observations. (1) The $\sim 5 \mathrm{~mm}$ deep radial artery was imaged with a higher SNR using LED arrays in comparison to the laser source, and (2) for deeper vessels in the subject's forearm, the laser at the $10 \mathrm{~Hz}$ frame rate had a slightly higher SNR compared to the LED arrays at $10 \mathrm{~Hz}$. In summary, due to the low power of LED arrays, a higher frame averaging is required to image deep tissue targets. LED-PAUS holds strong potential in point-of-care diagnosis of vascular diseases.

Author Contributions: Conceptualization: S.A., M.K.A.S. and S.-R.K.; Methodology: S.A., and K.J.; Software: S.A.; Hardware design: S.A.; Validation: S.A., M.K.A.S. and K.J.; Formal analysis: S.A. and M.K.A.S.; Investigation: S.-R.K.; Resources: S.-R.K.; Data curation: S.A. and M.K.A.S.; Writingoriginal draft preparation: S.A. and M.K.A.S.; Writing-review and editing: S.A., M.K.A.S., K.J., D.C.H., C.R.P., and S.-R.K.; Visualization: S.A., and K.J.; Supervision: S.-R.K.; Project administration: S.-R.K.; Funding acquisition, S.-R.K. All authors have read and agreed to the published version of the manuscript.

Funding: This project was funded by the NIH-NIBIB R00EB017729-04 (S.-R.K.), R21EB030370-01 (S.-R.K.) and support of Penn State Cancer Institute (S.-R.K.).

Institutional Review Board Statement: The human volunteer studies were conducted by following the internal imaging protocol of CYBERDYNE, INC (Rotterdam, The Netherlands) for healthyvolunteer imaging experiments, adhering to the guidelines of the Declaration of Helsinki.

Informed Consent Statement: Informed consent was obtained from all the subjects involved in this study.

Data Availability Statement: Data available on request from the authors.

Acknowledgments: We gratefully acknowledge the support from Gary Meyers and Eugene Gerber, for their help with the 3-D printing work and the machining of experimental setup parts.

Conflicts of Interest: M.K.A.S. is employed by CYBERDYNE, INC. The author(s) declare no potential conflicts of interest with respect to the research, authorship and/or publication of this article.

$\begin{array}{ll}\text { Abbreviations } \\ \text { PA } & \text { Photoacoustic } \\ \text { US } & \text { Ultrasound } \\ \text { PAI } & \text { Photoacoustic imaging } \\ \text { LED } & \text { Light-emitting diode } \\ \text { SNR } & \text { Signal to noise ratio } \\ \text { PRF } & \text { Pulse repetition frequency } \\ \text { OPO } & \text { Optical parametric oscillator } \\ \text { FWHM } & \text { Full width half maximum } \\ \text { MIP } & \text { Maximum intensity projection }\end{array}$




\section{References}

1. Norrving, B.; Kissela, B. The global burden of stroke and need for a continuum of care. Neurology 2013, 80, S5-S12. [CrossRef] [PubMed]

2. American Diabetes Association. Peripheral arterial disease in people with diabetes. Diabetes Care 2003, 26, 3333-3341. [CrossRef] [PubMed]

3. Stapleton, P.A.; James, M.E.; Goodwill, A.G.; Frisbee, J.C. Obesity and vascular dysfunction. Pathophysiology 2008, 15, 79-89. [CrossRef] [PubMed]

4. Zhang, Y.; Xia, H.; Wang, Y.; Chen, L.; Li, S.; Hussein, I.A.; Wu, Y.; Shang, Y.; Yao, S.; Du, R. The rate of missed diagnosis of lower-limb DVT by ultrasound amounts to $50 \%$ or so in patients without symptoms of DVT: A meta-analysis. Medicine 2019, 98, e17103. [CrossRef] [PubMed]

5. Pollak, A.W.; Norton, P.T.; Kramer, C.M. Multimodality imaging of lower extremity peripheral arterial disease: Current role and future directions. Circ. Cardiovasc. Imaging 2012, 5, 797-807. [CrossRef]

6. Ena, J.; Argente, C.R.; González-Sánchez, V.; Algado, N.; Verdú, G.; Lozano, T. Use of pocket pulse oximeters for detecting peripheral arterial disease in patients with diabetes mellitus. J. Diabetes Mellit. 2013, 3, 79-85. [CrossRef]

7. Ntziachristos, V.; Razansky, D. Molecular Imaging by Means of Multispectral Optoacoustic Tomography (MSOT). Chem. Rev. 2010, 110, 2783-2794. [CrossRef]

8. Bungart, B.L.; Lan, L.; Wang, P.; Li, R.; Koch, M.O.; Cheng, L.; Masterson, T.A.; Dundar, M.; Cheng, J.X. Photoacoustic tomography of intact human prostates and vascular texture analysis identify prostate cancer biopsy targets. Photoacoustics 2018, 11, 46-55. [CrossRef]

9. Hoelen, C.G.A.; De Mul, F.F.M.; Pongers, R.; Dekker, A. Three-dimensional photoacoustic imaging of blood vessels in tissue. Opt. Lett. 1998, 23, 648-650. [CrossRef]

10. Pan, D.; Pramanik, M.; Senpan, A.; Allen, J.S.; Zhang, H.; Wickline, S.A.; Wang, L.V.; Lanza, G.M. Molecular photoacoustic imaging of angiogenesis with integrin-targeted gold nanobeacons. FASEB J. 2010, 25, 875-882. [CrossRef]

11. Laufer, J.; Elwell, C.; Delpy, D.; Beard, P. In vitro measurements of absolute blood oxygen saturation using pulsed near-infrared photoacoustic spectroscopy: Accuracy and resolution. Phys. Med. Biol. 2005, 50, 4409. [CrossRef] [PubMed]

12. Lee, C.; Jeon, M.; Jeon, M.Y.; Kim, J.; Kim, C. In vitro photoacoustic measurement of hemoglobin oxygen saturation using a single pulsed broadband supercontinuum laser source. Appl. Opt. 2014, 53, 3884-3889. [CrossRef] [PubMed]

13. Lan, B.; Liu, W.; Wang, Y.-C.; Shi, J.; Li, Y.; Xu, S.; Sheng, H.; Zhou, Q.; Zou, J.; Hoffmann, U.; et al. High-speed widefield photoacoustic microscopy of small-animal hemodynamics. Biomed. Opt. Express 2018, 9, 4689-4701. [CrossRef] [PubMed]

14. Mallidi, S.; Luke, G.P.; Emelianov, S.Y. Photoacoustic imaging in cancer detection, diagnosis, and treatment guidance. Trends Biotechnol. 2011, 29, 213-221. [CrossRef] [PubMed]

15. Lv, J.; Li, S.; Zhang, J.; Duan, F.; Wu, Z.; Chen, R.; Chen, M.; Huang, S.; Ma, H.; Nie, L. In vivo photoacoustic im-aging dynamically monitors the structural and functional changes of ischemic stroke at a very early stage. Theranostics 2020, 10, 816. [CrossRef]

16. Kratkiewicz, K.; Manwar, R.; Rajabi-Estarabadi, A.; Fakhoury, J.; Meiliute, J.; Daveluy, S.; Mehregan, D.; Avanaki, K. Photoacoustic/Ultrasound/Optical Coherence Tomography Evaluation of Melanoma Lesion and Healthy Skin in a Swine Model. Sensors 2019, 19, 2815. [CrossRef]

17. Guggenheim, J.A.; Allen, T.J.; Plumb, A.; Zhang, E.; Rodriguez-Justo, M.; Punwani, S.; Beard, P.C. Photoacoustic imaging of human lymph nodes with endogenous lipid and hemoglobin contrast. J. Biomed. Opt. 2015, 20, 050504. [CrossRef]

18. Kole, A.; Cao, Y.; Hui, J.; Bolad, I.A.; Alloosh, M.; Cheng, J.-X.; Sturek, M. Comparative Quantification of Arterial Lipid by Intravascular Photoacoustic-Ultrasound Imaging and Near-Infrared Spectroscopy-Intravascular Ultrasound. J. Cardiovasc. Transl. Res. 2018, 12, 211-220. [CrossRef]

19. Xu, Z.; Zhu, Q.; Wang, L.V. In vivo photoacoustic tomography of mouse cerebral edema induced by cold injury. J. Biomed. Opt. 2011, 16, 066020. [CrossRef]

20. Yao, D.K.; Maslov, K.; Shung, K.K.; Zhou, Q.; Wang, L.V. In vivo label-free photoacoustic microscopy of cell nuclei by excitation of DNA and RNA. Opt. Lett. 2010, 35, 4139-4141. [CrossRef]

21. Weber, J.; Beard, P.C.; Bohndiek, S.E. Contrast agents for molecular photoacoustic imaging. Nat. Methods 2016, 13, 639-650 [CrossRef] [PubMed]

22. Luke, G.P.; Yeager, D.; Emelianov, S. Biomedical Applications of Photoacoustic Imaging with Exogenous Contrast Agents. Ann. Biomed. Eng. 2012, 40, 422-437. [CrossRef] [PubMed]

23. Dragulescu-Andrasi, A.; Kothapalli, S.R.; Tikhomirov, G.A.; Rao, J.; Gambhir, S.S. Activatable oligomerizable imaging agents for photoacoustic imaging of furin-like activity in living subjects. J. Am. Chem. Soc. 2013, 135, 11015-11022. [CrossRef] [PubMed]

24. Cheng, K.; Kothapalli, S.-R.; Liu, H.; Koh, A.L.; Jokerst, J.V.; Jiang, H.; Yang, M.; Li, J.; Levi, J.; Wu, J.C.; et al. Construction and Validation of Nano Gold Tripods for Molecular Imaging of Living Subjects. J. Am. Chem. Soc. 2014, 136, 3560-3571. [CrossRef] [PubMed]

25. Zemp, R.J.; Li, L.; Wang, L.V. Photoacoustic Imaging of Gene Expression in Small Animals in Vivo. In Photoacoustic Imaging and Spectroscopy; CRC Press: Boca Raton, FL, USA, 2017; pp. 363-370.

26. Ahn, S.; Kang, J.; Kim, P.; Lee, G.; Jeong, E.; Jung, W.; Park, M.; Song, T.-K. Smartphone-based portable ultrasound imaging system: Prototype implementation and evaluation. In Proceedings of the 2015 IEEE International Ultrasonics Symposium (IUS), Taipei, Taiwan, 21-24 October 2015. 
27. Jawahar, D.; Rachamalla, H.R.; Rafalowski, A.; Ilkhani, R.; Bharathan, T.; Anandarao, N.; Jawahar, D. Pulse oximetry in the evaluation of peripheral vascular disease. Angiology 1997, 48, 721-724. [CrossRef] [PubMed]

28. Regensburger, A.P.; Fonteyne, L.M.; Jüngert, J.; Wagner, A.L.; Gerhalter, T.; Nagel, A.M.; Heiss, R.; Flenkenthaler, F.; Qurashi, M.; Neurath, M.F.; et al. Detection of collagens by multispectral optoacoustic tomography as an imaging biomarker for Duchenne muscular dystrophy. Nat. Med. 2019, 25, 1905-1915. [CrossRef]

29. Kothapalli, S.-R.; Sonn, G.A.; Choe, J.W.; Nikoozadeh, A.; Bhuyan, A.; Park, K.K.; Cristman, P.; Fan, R.; Moini, A.; Lee, B.C.; et al Simultaneous transrectal ultrasound and photoacoustic human prostate imaging. Sci. Transl. Med. 2019, 11, eaav2169. [CrossRef]

30. Dutta, R.; Mandal, S.; Lin, H.-C.A.; Raz, T.; Kind, A.; Schnieke, A.; Razansky, D. Brilliant cresyl blue enhanced optoacoustic imaging enables non-destructive imaging of mammalian ovarian follicles for artificial reproduction. J. R. Soc. Interface 2020, 17, 20200776. [CrossRef]

31. Oraevsky, A.A.; Clingman, B.; Zalev, J.; Stavros, A.T.; Yang, W.T.; Parikh, J.R. Clinical optoacoustic imaging combined with ultrasound for coregistered functional and anatomical mapping of breast tumors. Photoacoustics 2018, 12, 30-45. [CrossRef]

32. Allen, T.J.; Beard, P.C. Pulsed near-infrared laser diode excitation system for biomedical photoacoustic imaging. Opt. Lett. 2006, 31, 3462-3464. [CrossRef]

33. Yao, Q.; Ding, Y.; Liu, G.; Zeng, L. Low-cost photoacoustic imaging systems based on laser diode and light-emitting diode excitation. J. Innov. Opt. Health Sci. 2017, 10, 1730003. [CrossRef]

34. Kolkman, R.G.M.; Steenbergen, W.; Van Leeuwen, T.G. In vivo photoacoustic imaging of blood vessels with a pulsed laser diode. Lasers Med Sci. 2006, 21, 134-139. [CrossRef] [PubMed]

35. Upputuri, P.K.; Pramanik, M. Fast photoacoustic imaging systems using pulsed laser diodes: A review. Biomed. Eng. Lett. 2018, 8, 167-181. [CrossRef] [PubMed]

36. Dangi, A.; Agrawal, S.; Kothapalli, S.R. Lithium niobate-based transparent ultrasound transducers for photoacoustic imaging. Opt. Lett. 2019, 44, 5326-5329. [CrossRef]

37. Dangi, A.; Cheng, C.Y.; Agrawal, S.; Tiwari, S.; Datta, G.R.; Benoit, R.R.; Pratap, R.; Trolier-McKinstry, S.; Kothapalli, S.-R. A Photoacoustic Imaging Device Using Piezoelectric Micromachined Ultrasound Transducers (PMUTs). IEEE Trans. Ultrason. Ferroelectr. Freq. Control 2020, 67, 801-809. [CrossRef]

38. Chen, M.-C.; Perez, A.P.; Kothapalli, S.-R.; Cathelin, P.; Cathelin, A.; Gambhir, S.S.; Murmann, B. A Pixel Pitch-Matched Ultrasound Receiver for 3-D Photoacoustic Imaging With Integrated Delta-Sigma Beamformer in 28-nm UTBB FD-SOI. IEEE J. Solid-State Circuits 2017, 52, 2843-2856. [CrossRef]

39. Kothapalli, S.R.; Ma, T.J.; Vaithilingam, S.; Oralkan, Ö.; Khuri-Yakub, B.T.; Gambhir, S.S. Deep tissue photoacous-tic imaging using a miniaturized 2-D capacitive micromachined ultrasonic transducer array. IEEE Trans. Biomed. Eng. 2012, 59, 1199-1204. [CrossRef]

40. Liu, S.; Tang, K.; Feng, X.; Jin, H.; Gao, F.; Zheng, Y. Toward Wearable Healthcare: A Miniaturized 3D Imager With Coherent Frequency-Domain Photoacoustics. IEEE Trans. Biomed. Circuits Syst. 2019, 13, 1417-1424. [CrossRef]

41. Dangi, A.; Agrawal, S.; Datta, G.R.; Srinivasan, V.; Kothapalli, S.R. Towards a Low-Cost and Portable Photoacoustic Microscope for Point-of-Care and Wearable Applications. IEEE Sens. J. 2020, 20, 6881-6888. [CrossRef]

42. Hariri, A.; Fatima, A.; Mohammadian, N.; Mahmoodkalayeh, S.; Ansari, M.A.; Bely, N.; Avanaki, M.R. Development of low-cost photoacoustic imaging systems using very low-energy pulsed laser diodes. J. Biomed. Opt. 2017, 22, 075001. [CrossRef]

43. Zhong, H.; Duan, T.; Lan, H.; Zhou, M.; Gao, F. Review of Low-Cost Photoacoustic Sensing and Imaging Based on Laser Diode and Light-Emitting Diode. Sensors 2018, 18, 2264. [CrossRef] [PubMed]

44. Allen, T.J.; Beard, P.C. High power visible light emitting diodes as pulsed excitation sources for biomedical photoacoustics. Biomed. Opt. Express 2016, 7, 1260-1270. [CrossRef] [PubMed]

45. Hansen, R.S. Using high-power light emitting diodes for photoacoustic imaging. In Proceedings of the Medical Imaging 2011: Ultrasonic Imaging, Tomography, and Therapy, Lake Buena Vista (Orlando), FL, USA, 13-14 February 2011; International Society for Optics and Photonics: Bellingham, WA, USA, 2011; Volume 7968, p. 79680A.

46. Dai, X.; Yang, H.; Jiang, H. In vivo photoacoustic imaging of vasculature with a low-cost miniature light emitting diode excitation. Opt. Lett. 2017, 42, 1456-1459. [CrossRef] [PubMed]

47. Zhu, Y.; Feng, T.; Cheng, Q.; Wang, X.; Du, S.; Sato, N.; Yuan, J.; Kuniyil Ajith Singh, M. Towards Clinical Translation of LED-Based Photoacoustic Imaging: A Review. Sensors 2020, 20, 2484. [CrossRef] [PubMed]

48. Zhu, Y.; Xu, G.; Yuan, J.; Jo, J.; Gandikota, G.; Demirci, H.; Agano, T.; Sato, N.; Shigeta, Y.; Wang, X. Light emitting diodes based photoacoustic imaging and potential clinical applications. Sci. Rep. 2018, 8, 9885. [CrossRef] [PubMed]

49. Agrawal, S.; Fadden, C.; Dangi, A.; Yang, X.; AlBahrani, H.; Frings, N.; Zadi, S.H.; Kothapalli, S.-R. Light-Emitting-Diode-Based Multispectral Photoacoustic Computed Tomography System. Sensors 2019, 19, 4861. [CrossRef] [PubMed]

50. Joseph, F.K.; Boink, Y.E.; Dantuma, M.; Ajith, S.M.; Manohar, S.; Steenbergen, W. Tomographic imaging with an ultrasound and LED-based photoacoustic system. Biomed. Opt. Express 2020, 11, 2152-2165. [CrossRef]

51. Zhu, Y.; Lu, X.; Dong, X.; Yuan, J.; Fabiilli, M.L.; Wang, X. LED-based photoacoustic imaging for monitoring angiogenesis in fibrin scaffolds. Tissue Eng. Part C: Methods 2019, 25, 523-531. [CrossRef]

52. Xavierselvan, M.; Singh, M.K.A.; Mallidi, S. In Vivo Tumor Vascular Imaging with Light Emitting Diode-Based Photoacoustic Imaging System. Sensors 2020, 20, 4503. [CrossRef] 
53. Jo, J.; Xu, G.; Zhu, Y.; Burton, M.; Sarazin, J.; Schiopu, E.; Gandikota, G.; Wang, X. Detecting joint inflammation by an LED-based photoacoustic imaging system: A feasibility study. J. Biomed. Opt. 2018, 23, 110501-110504. [CrossRef]

54. Maneas, E.; Aughwane, R.; Huynh, N.; Xia, W.; Ansari, R.; Kuniyil Ajith Singh, M.; Hutchinson, J.C.; Sebire, N.J.; Arthurs, O.J.; Deprest, J.; et al. Photoacoustic imaging of the human placental vasculature. J. Biophotonics 2020, 13, e201900167. [CrossRef] [PubMed]

55. Xia, W.; Maneas, E.; Huynh, N.T.; Singh, M.K.A.; Brown, N.M.; Ourselin, S.; Gilbert-Kawai, E.; West, S.J.; Desjardins, A.E. Imaging of human peripheral blood vessels during cuff occlusion with a compact LED-based photoacoustic and ultrasound system. In Photons Plus Ultrasound: Imaging and Sensing; International Society for Optics and Photonics: Bellingham, WA, USA, 2019; Volume 10878, p. 1087804.

56. Agano, T.; Singh, M.K.A.; Nagaoka, R.; Awazu, K. Effect of light pulse width on frequency characteristics of photoacoustic signal-An experimental study using a pulse-width tunable LED-based photoacoustic imaging system. Int. J. Eng. Technol. 2018, 7, 4300-4303.

57. Jaeger, M.; Schüpbach, S.; Gertsch, A.; Kitz, M.; Frenz, M. Fourier reconstruction in optoacoustic imaging using truncated regularized inverse k -space interpolation. Inverse Probl. 2007, 23, S51-S63. [CrossRef]

58. Marquez, G.; Wang, L.V.; Lin, S.-P.; Schwartz, J.A.; Thomsen, S.L. Anisotropy in the absorption and scattering spectra of chicken breast tissue. Appl. Opt. 1998, 37, 798-804. [CrossRef]

59. American National Standards Institute. American National Standard for Safe Use of Lasers; Laser Institute of America: Orlando, FL, USA, 2007.

60. Anas, E.M.A.; Zhang, H.K.; Kang, J.; Boctor, E. Enabling fast and high quality LED photoacoustic imaging: A recurrent neural networks based approach. Biomed. Opt. Express 2018, 9, 3852-3866. [CrossRef]

61. Farnia, P.; Najafzadeh, E.; Hariri, A.; Lavasani, S.N.; Makkiabadi, B.; Ahmadian, A.; Jokerst, J.V. Dictionary learning technique enhances signal in LED-based photoacoustic imaging. Biomed. Opt. Express 2020, 11, 2533-2547. [CrossRef]

62. Johnstonbaugh, K.; Agrawal, S.; Durairaj, D.A.; Fadden, C.; Dangi, A.; Karri, S.P.K.; Kothapalli, S.R. A deep learning approach to photoacoustic wavefront localization in deep-tissue medium. IEEE Trans. Ultrason. Ferroelectr. Freq. Control 2020, 67, $2649-2659$. [CrossRef] 